\title{
SnackNTM: An Open-Source Software for Sanger Sequencing-based Identification of Nontuberculous Mycobacterial Species
}

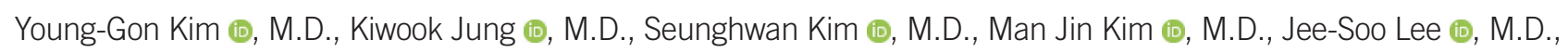
Sung-Sup Park (i), M.D., Ph.D., and Moon-Woo Seong iㅣ, M.D., Ph.D.

Department of Laboratory Medicine, Seoul National University Hospital, Seoul National University College of Medicine, Seoul, Korea

Background: Sequence-based identification is one of the most effective methods for species-level identification of nontuberculous mycobacteria (NTM). However, it is time-consuming because of the bioinformatics processes involved, including sequence trimming, consensus sequence generation, and public database searches. We developed a simple and fully automated software that enabled species-level identification of NTM from trace files, SnackNTM (https://github.com/Young-gonKim/SnackNTM).

Methods: JAVA programing language was used for software development. The SnackNTM diagnostic algorithm utilized 16S rRNA gene sequences, according to the Clinical \& Laboratory Standards Institute guidelines, and an $r p o B$ gene region was adjunctively utilized to narrow down the species. The software performance was validated using trace files of 234 clinical cases, comprising 217 consecutive cases and 17 additionally selected cases of unique species.

Results: SnackNTM could analyze multiple cases at once, and all the bioinformatics processes required for sequence-based NTM identification were automatically performed with a single mouse click. SnackNTM successfully identified 95.9\% (208/217) of consecutive clinical cases, and the results showed 99.0\% (206/208) agreement with manual classification results. SnackNTM successfully identified all 17 cases of unique species. In a processing time comparison test, the analysis and reporting of 30 cases, which took 150 minutes manually, took only 40 minutes with SnackNTM.

Conclusions: SnackNTM is expected to reduce the workload for NTM identification, especially in clinical laboratories that process large numbers of cases.

Key Words: Nontuberculous mycobacteria, NTM identification, SnackNTM, 16S rRNA gene, $r p o B$
Received: February 24, 2021

Revision received: April 23, 2021

Accepted: September 9, 2021

\section{Corresponding author:}

Moon-Woo Seong, M.D., Ph.D.

Department of Laboratory Medicine, Seoul National University Hospital, Seoul National University College of Medicine, 101 Daehak-ro, Jongno-gu, Seoul 03080, Korea Tel: +82-2-2072-4180

Fax: +82-2-747-0359

E-mail: mwseong@snu.ac.kr

\section{INTRODUCTION}

The incidence of nontuberculous mycobacterial infections has increased worldwide, posing several challenges for clinicians and clinical laboratories $[1,2]$. As the clinical relevance of infection varies widely with the species of nontuberculous mycobacteria (NTM), species-level identification is fundamental for proper patient management [3-5]. The differential susceptibility of different species to various antibiotics renders correct species identification even more important [6, 7]. However, species identification is not straightforward as there are more than 190 validly published species in the genus Mycobacterium [8]. The Clinical and Laboratory Standards Institute (CLSI) guidelines suggest the use of both phenotypic methods, such as matrix- 
assisted laser desorption ionization-time of flight mass spectrometry (MALDI-TOF MS), and genotypic methods, such as line probe assays, for mycobacterial identification [9]. According to the CLSI guidelines, nucleic acid amplification and sequencing (usually, Sanger sequencing) is one of the most comprehensive and definitive methods for mycobacterial identification.

Sequence-based species identification involves several bioinformatics processes, including the reviewing and trimming of trace files, the generation of consensus sequences of forward and reverse traces, and searching public databases using tools such as Basic Local Alignment Search Tool (BLAST) (https:// blast.ncbi.nlm.nih.gov/Blast.cgi). BLAST results usually include multiple species and should be filtered according to predefined criteria. These processes require time and effort, especially when multiple genome regions from multiple cases are to be evaluated. The sequences submitted to public databases lack validation in terms of sequence quality and contemporariness of the mycobacterial taxonomic classification [10-12].

We developed a software for automated batch processing of Sanger sequence data for NTM identification and validated it using clinical data. To the best of our knowledge, SnackNTM is the first software for automated NTM identification based on Sanger sequencing.

\section{MATERIALS AND METHODS}

\section{Study design}

This study was approved by the Institutional Review Board of Seoul National University Hospital (SNUH), Seoul, Korea. This study comprised two parts: software development and validation, both of which were performed at SNUH. Software was developed between June 2019 and December 2019. Validation was performed as a retrospective study utilizing sequencing trace files stored in the SNUH repository between July 2020 and August 2020.

\section{Software development}

Java Development Kit 8 (Oracle, CA, USA) and its component JavaFX were used to develop a graphical user interface-based software. BioJava Legacy (https://github.com/biojava/biojavalegacy) was used to read sequence trace files (.ab1 files). Basic functions required for the handling of sequence files, including reading trace files, automatic trimming, and the alignment algorithm, were adopted from our previous work [13].

The workflow of SnackNTM is summarized in Fig. 1. SnackNTM reads multiple trace files at once and allocates them according

\section{Read trace files}

All files in a batch at one time

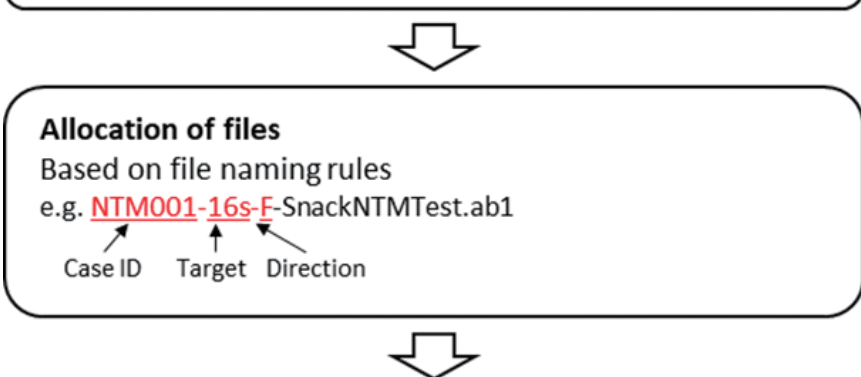

Automatic trimming of all traces

Based on quality score

\section{ए}

\section{Alignment \& scoring}

For each case

- For each target region

- For each species in reference database

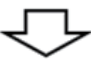

Ranking of species based on percent identity scores

For each case

- For each target region

\section{Interpretation of results}

For each case

- Combination of results from 2 target regions

Fig. 1. Schematic representation of the workflow of SnackNTM. SnackNTM reads multiple trace files at once and allocates them according to the cases and target regions. After automatic trimming is performed for all traces, input sequences are iteratively aligned to all locally stored reference sequences. The percent identity score is then calculated for every alignment and the species are sorted according to their scores. The results for the two target regions are combined to produce the final identification result.

to the case and target region. The maximum number of cases successfully processed during the validation was 217 , with 868 sequence trace files. After the trace files are trimmed, the input sequences are iteratively aligned with locally stored reference sequences. The percent identity score is then calculated for every alignment, and the species are sorted according to their scores. Species that satisfy the identification criteria described in the next subsection are output as the identification results. 
Kim YG, et al.

SnackNTM software for NTM identification

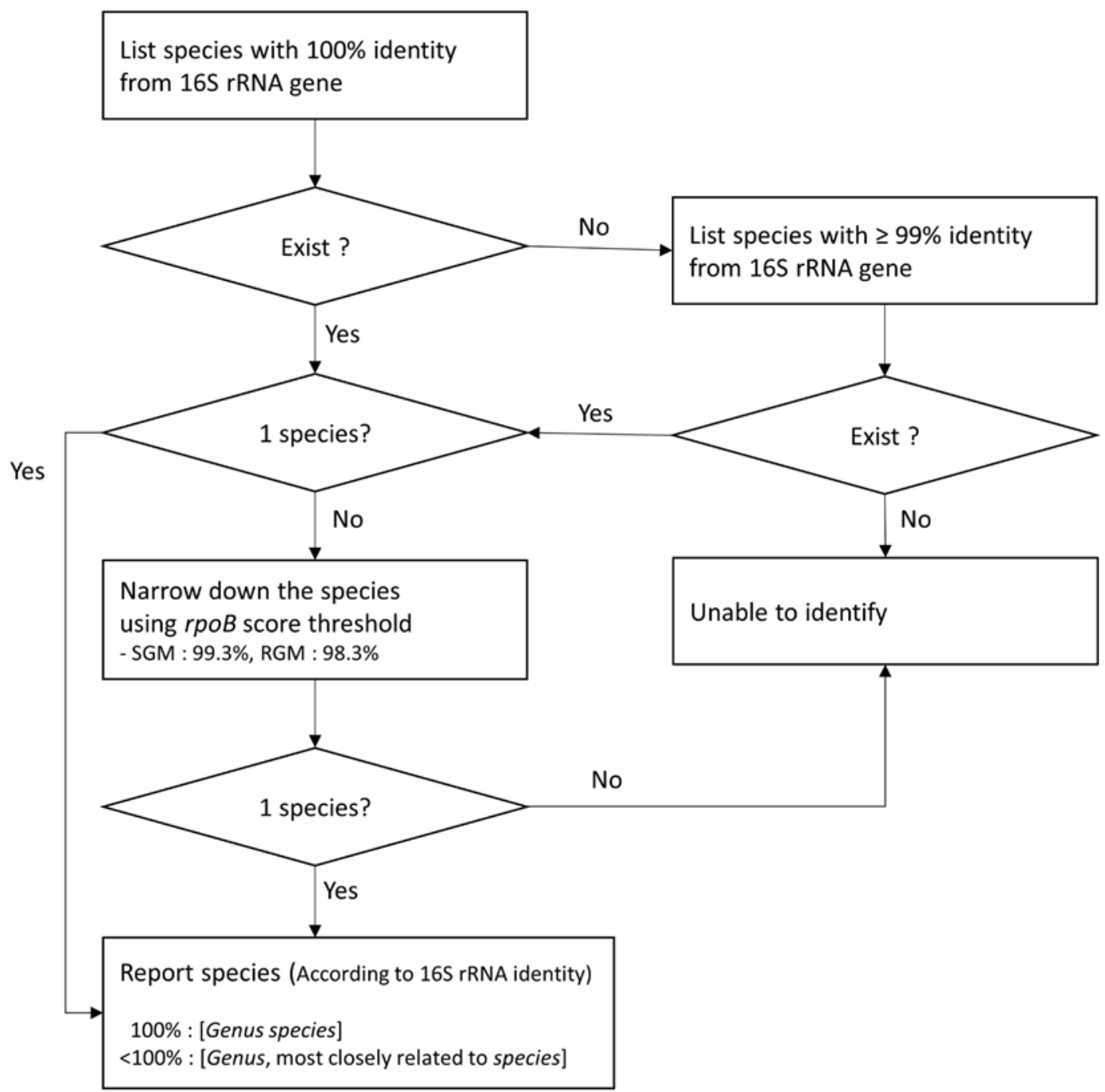

Fig. 2. Identification criteria employed in SnackNTM. Species with 100\% identity based on 16S rRNA gene sequences, if available, are selected for further analysis. If not, species with $\geq 99 \%$ identity based on $16 S$ rRNA gene sequences are selected. When multiple species satisfy the 16S rRNA gene sequence criteria, rpoB sequence analysis results are utilized to narrow down the species. The cutoff values used in rpoB sequence analysis were $99.3 \%$ for slowgrowing mycobacteria and $98.3 \%$ for rapidly growing mycobacteria, according to a previous study [5].

Abbreviations: SGM, slowgrowing mycobacteria; RGM, rapidly growing mycobacteria.

\section{Target regions and reference sequences}

The 5' end of the 16S rRNA gene (approximately 500 bp) and part of $r p o B$ were amplified using reported primers [14]. The primers for the 16S rRNA gene were 285-F (5'-GAGAGTTTGATCCTGGCTCAG-3') and 247-R (5'-TTTCACGAACAACGCGACAA-3'). The primers for rpoB were Myco-F (5'-GGCAAGGTCACCCCGAAGGG-3') and Myco-R (5'-AGCGGCTGCTGGGTGATCATC-3'). Reference sequences of the targeted regions of mycobacterial species were curated from GenBank (National Center for Biotechnology Information; https://www.ncbi.nlm.nih. gov/genbank/, accessed on February 24, 2021) and stored lo- cally. When available, sequences of type strains were selected. The number of curated species, including subspecies of four species, was 212 for the $16 \mathrm{~S}$ rRNA gene and 174 for rpoB. The list of reference sequences can be found at https://github.com/ Young-gonKim/SnackNTM/tree/batch/reference (accessed on February 24, 2021).

\section{Identification criteria}

The identification process used in SnackNTM is shown in Fig. 2. The interpretative criteria used at SNUH were adopted. According to the CLSI guidelines MM18-A, a threshold of $99.0 \%$ se- 
quence identity for the 16S rRNA gene was used [11]. However, species with $100 \%$ identity were preferentially considered. When identification based on the 16S rRNA gene failed to narrow down to a single species, rpoB sequencing results were additionally used. As the CLSI guidelines do not define sequence identity cutoff values for rpoB, they were adopted from a previous study, as follows: $99.3 \%$ for slowly growing mycobacteria and $98.3 \%$ for rapidly growing mycobacteria [5]. Cases with no species showing $>99 \%$ identity for the 16S rRNA gene and those with results indicative of multiple species even after the incorporation of $r p o B$ sequence data were reported as unidentifiable. Although subspecies-level reference sequences of four species were included in the local database, subspecies level identification was provided only for Mycobacterium abscessus, whose subspecies have a distinctive antimicrobial susceptibility [15].

\section{Validation using consecutive case data}

Trace files of 217 consecutive cases, which were produced from NTM identification tests performed between July 2019 and August 2019, were reanalyzed using both SnackNTM and a manual identification process, in which sequences were trimmed using the default trimming function of Sequencher (Gene Codes, Ann Arbor, MI, USA). The consensus sequences of forward and reverse traces thus generated were fed to locally installed BLAST to search the locally stored reference sequences. The same sets of reference sequences were used in SnackNTM and in manual identification. The results from the two methods were compared, and the percentage of agreement was calculated.

\section{Validation using unique species data}

Since the consecutive case data contained a limited number of species (11 species from 217 cases), to utilize more species for validation, we performed additional tests using selected cases with unique species. Unique species data comprised 11 cases selected from the consecutive case data described above and 17 additional cases selected from the SNUH repository. Case selection was based on reported identification results obtained at SNUH between May 2017 and October 2019. Among cases with the same reported species, the case with the earliest test date was selected. The 28 cases with unique species data finally included were analyzed using SnackNTM and EzBioCloud (https://www.ezbiocloud.net/identify), which utilizes 16S rRNA gene sequences for bacterial identification. The results were compared, and the percentage of agreement was calculated.

\section{Comparison of the processing time between SnackNTM and} manual identification

Thirty cases were selected from the consecutive case data and two researchers, $\mathrm{KJ}$ and SK, analyzed the selected cases using SnackNTM and manual identification. The time required for both analyses by both researchers was measured, and the mean processing times for the analyses were compared.

\section{RESULTS}

\section{SnackNTM software}

A simple windowed application was developed for NTM identification. Detailed instructions on how to use SnackNTM are provided in a movie clip that can be found on https://github.com/ Young-gonKim/SnackNTM (accessed on February 24, 2021). The status of SnackNTM after completion of the initial analysis is shown in Fig. 3. In this example, 120 trace files generated from 30 cases were selected at once and allocated automatically to proper positions according to the file naming rules that utilize case ID, target region, and read direction (forward vs. reverse) contained in the file names. Clicking the button "Run (All samples)" initiates the analysis, which takes a few seconds per case. As the trace files occasionally contain base-calling errors, especially in the trace ends, the traces should be reviewed after the initial automatic analysis. When an error is found, it can be fixed using the "Edit base" function, and when the conclusion contains unexpected results, such as multiple species or no species, the "Edit trimming" function can be used to increase or decrease the range of sequences to be included in the analysis. The identification results can be exported either in the tabseparated value format or as an excel file using the "Export" function in the data tab.

\section{Validation using consecutive case data}

The SnackNTM and manual identification results of all cases are listed in Table 1, and a summary of the species identified by SnackNTM is presented in Table 2. Among 217 cases, 208 (95.9\%) were successfully identified to a single species. The most frequently identified species were M. avium (80/217, 36.9\%), M. intracellulare (74/217, 34.1\%), M. abscessus subsp. abscessus $(27 / 217,12.4 \%)$, and M. massiliense (16/217, 7.4\%). Nine $(4.1 \%)$ cases were unidentifiable using SnackNTM because the results indicated multiple species. These cases were also unidentifiable by manual identification for the same reason.

For case NTM095, SnackNTM yielded unidentifiable results because the identification process resulted in two species, $M$. kan- 


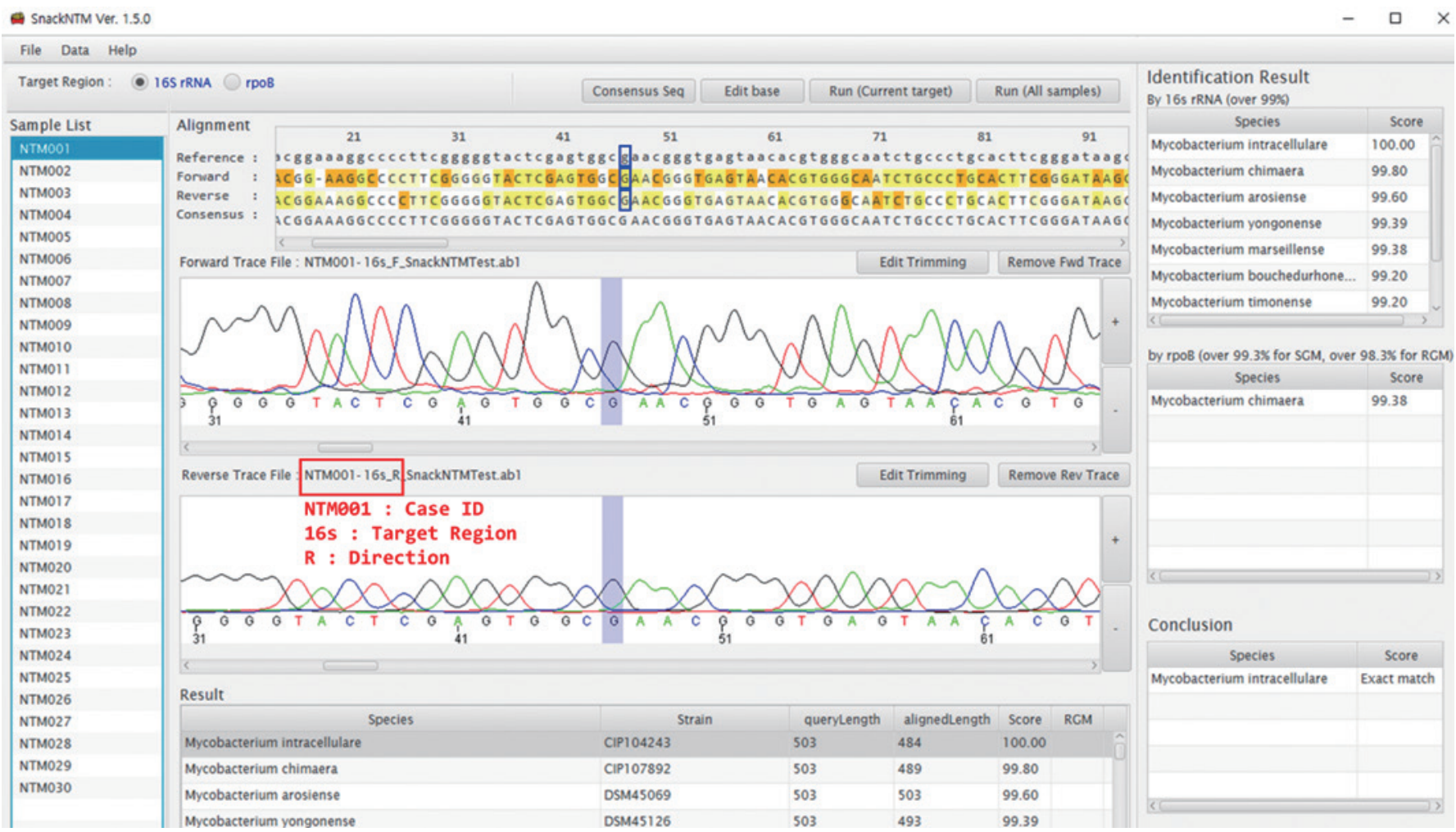

Fig. 3. Snapshot of SnackNTM after initial analysis. Trace files from all cases in a batch are loaded at once and allocated to their positions in a program according to a predefined file naming rule. The alignment results are browsed in the "Alignment" panel, and chromatograms appear below according to mouse clicks on the "Alignment" panel. Miscalled bases can be corrected by clicking the "Edit base" button. In the "Identification Result" panel, species that satisfy the criteria of each target region are shown. Final results are shown in the "Conclusion" panel.

sasii and M. gastri, which cannot be distinguished based on the 16S rRNA gene sequences [10, 11, 16, 17]. However, in cases NTM003 and NTM076, M. kansasii was correctly identified based on the rpoB sequence. The rpoB sequence of NTM095 could not be utilized because of poor trace quality.

In another unidentifiable case, NTM086, the identification results were $M$. chimaera and $M$. intracellulare. $M$. chimaera is often misidentified as $M$. intracellulare because the 16S rRNA gene sequences of both species are identical, except for one nucleotide at position 403 [18]. For NTM086, the results showed a percent identity between $99 \%$ and $100 \%$ for M. chimaera and $M$. intracellulare based on the 16S rRNA gene, and both species passed the threshold of rpoB sequence analysis. In this case, the nucleotide at position 403 was C, indicating a higher likelihood of it being $M$. chimaera. $M$. chimaera was correctly identified in NTM162 based on 100\% 16S rRNA gene sequence identity [19].

Among the 208 cases that were identified as a single species by both SnackNTM and manual identification, 206 cases (99.0\%) showed agreement. The discrepancy in the remaining two cases (NTM039 and NTM137) was caused by a difference in the trimming procedure between SnackNTM and Sequencher. In both cases, a longer portion of the trace was trimmed out in manual identification, leading to the utilization of shorter input sequences.

\section{Validation using unique species data}

The results of identification using unique species data are described in Table 3. The SnackNTM results agreed with the manual identification results in all 28 cases. The EzBioCloud results agreed with the SnackNTM results in 23 cases (82.1\%). Discrepancies between SnackNTM and EzBioCloud results were due to the utilization of $r p o B$ sequences in SnackNTM $(4 / 5,80.0 \%)$ and differences in the set of reference sequences $(1 / 5,20.0 \%)$.

Comparison of the processing time between SnackNTM and manual identification

SnackNTM analysis and manual identification of the 30 cases selected for processing time comparison took 40 minutes and 


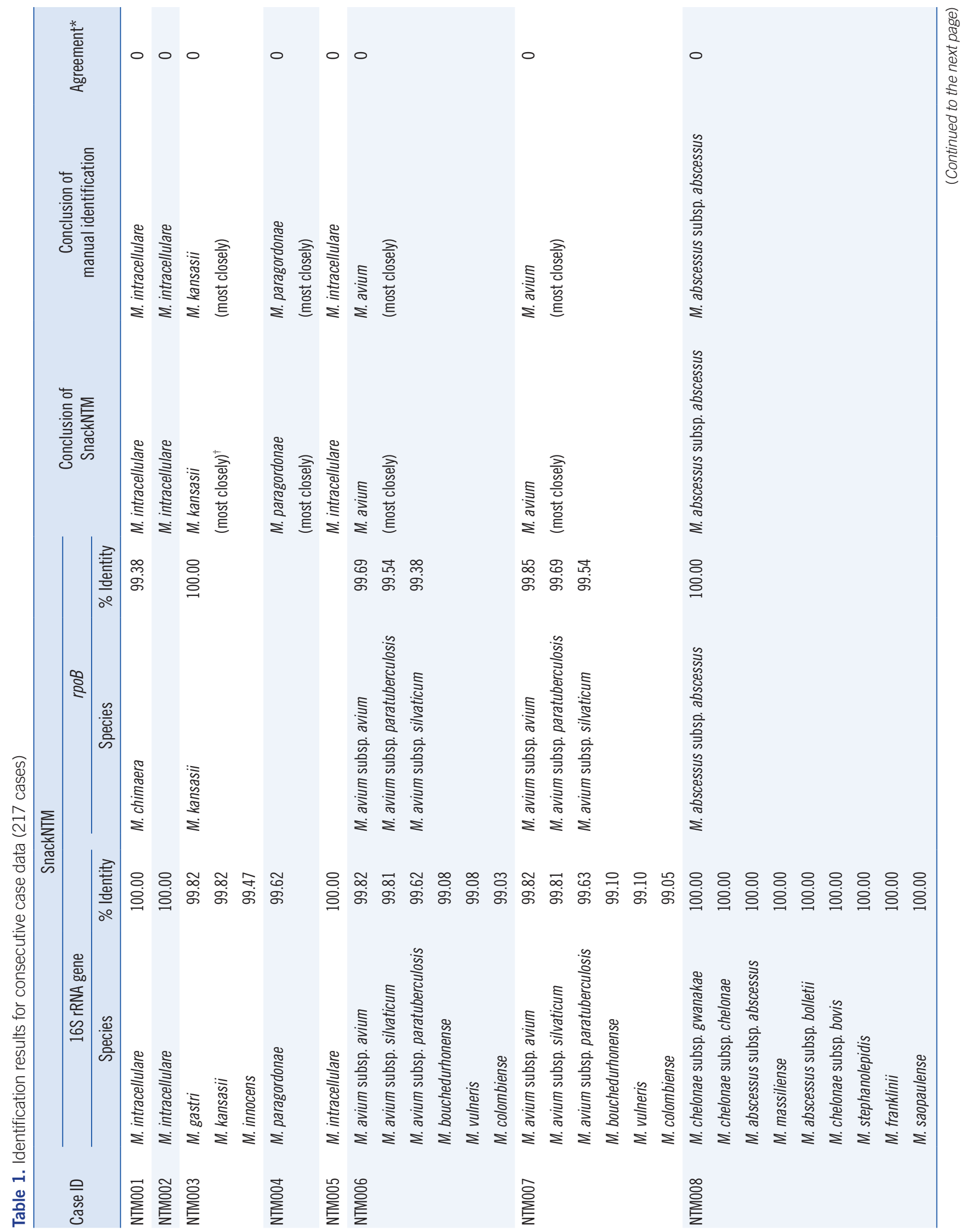


Kim YG, et al.

SnackNTM software for NTM identification

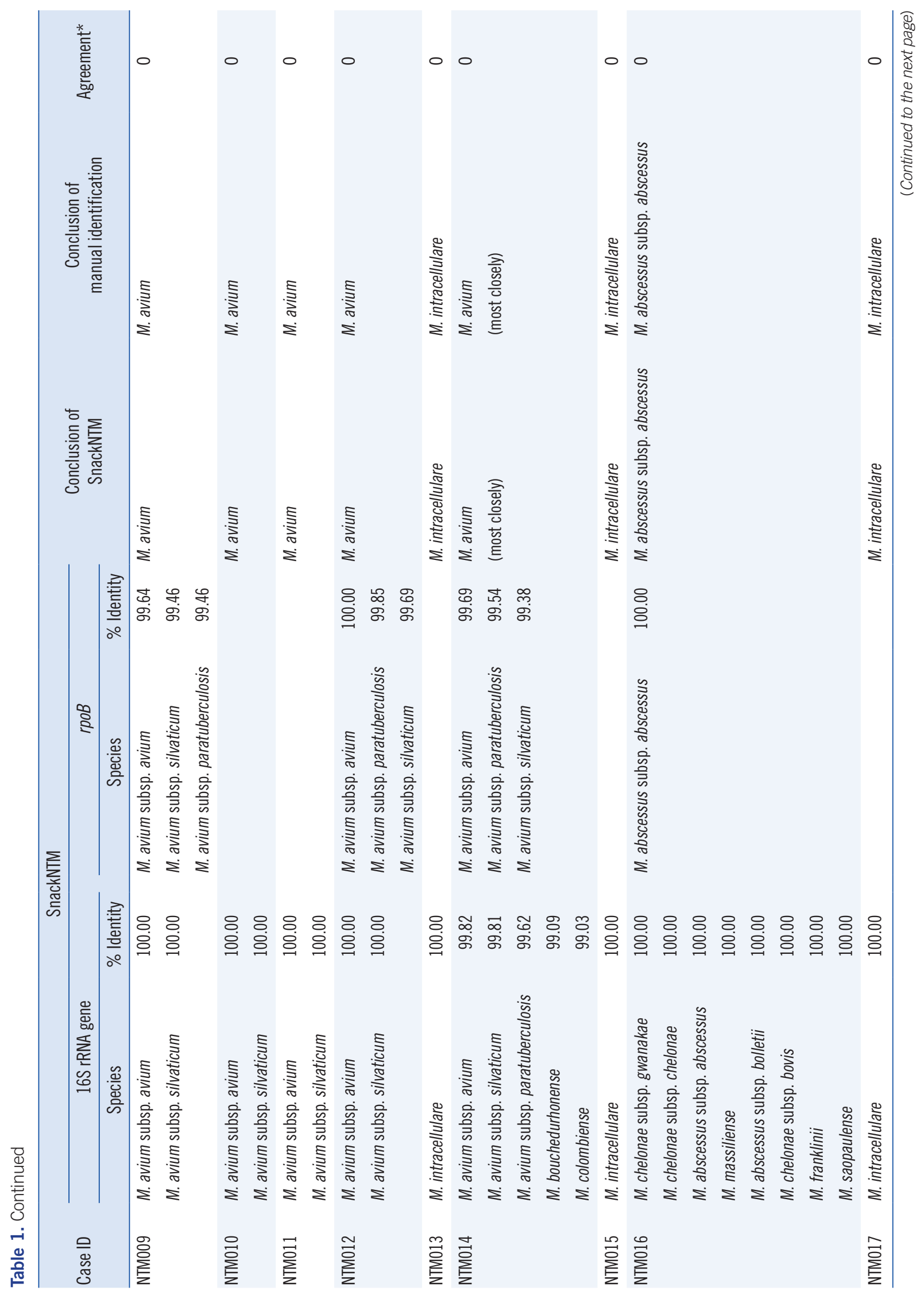




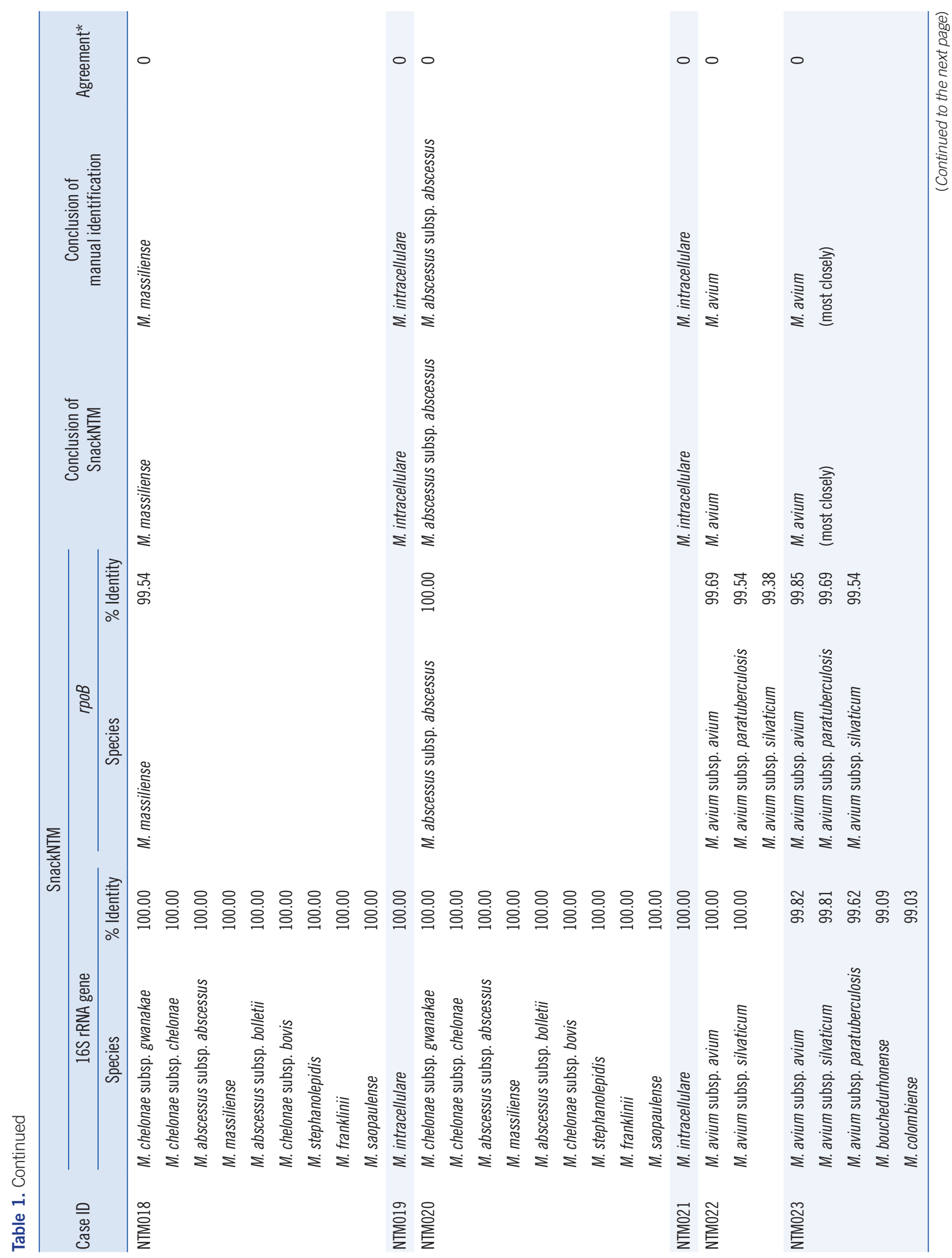


Kim YG, et al.

SnackNTM software for NTM identification

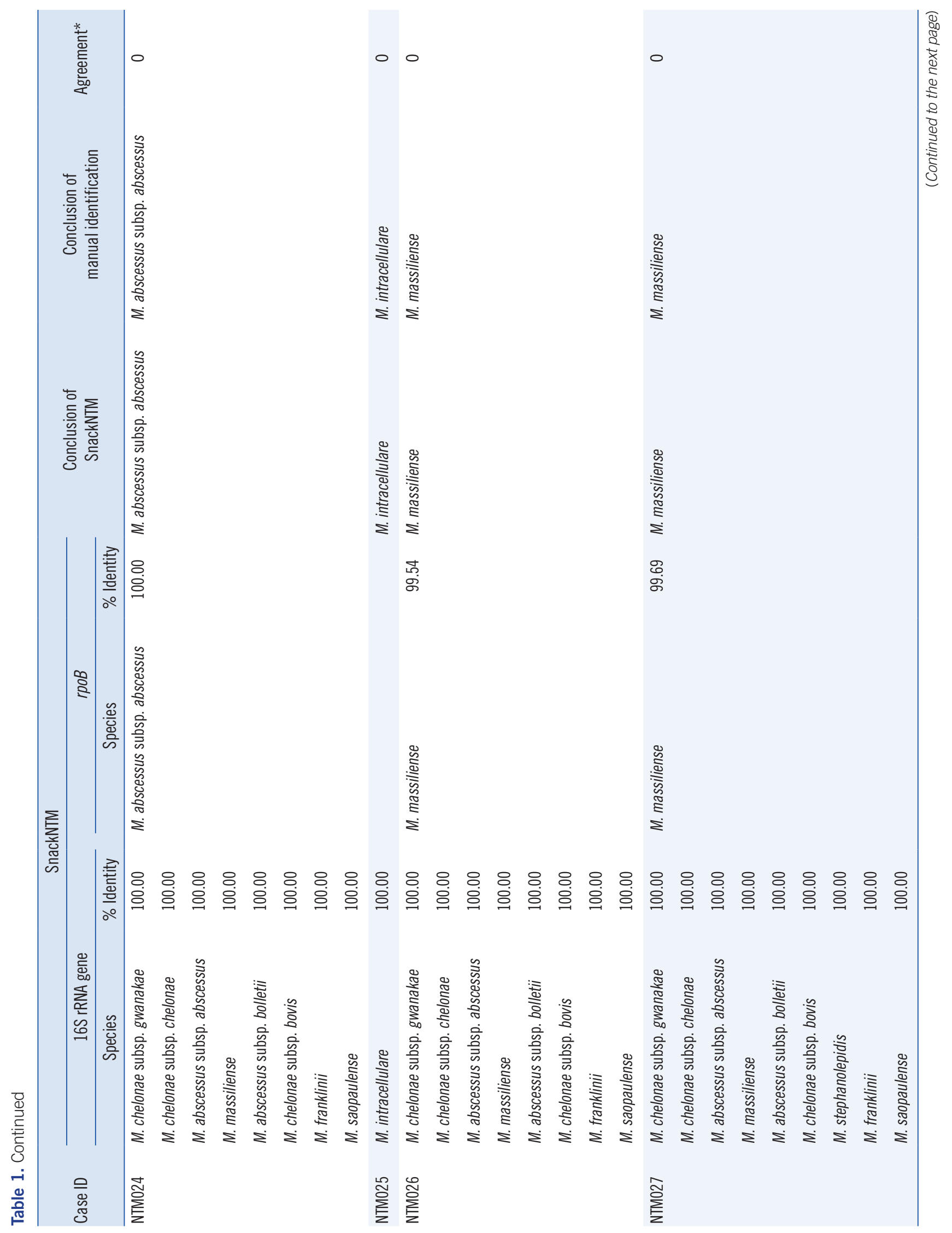




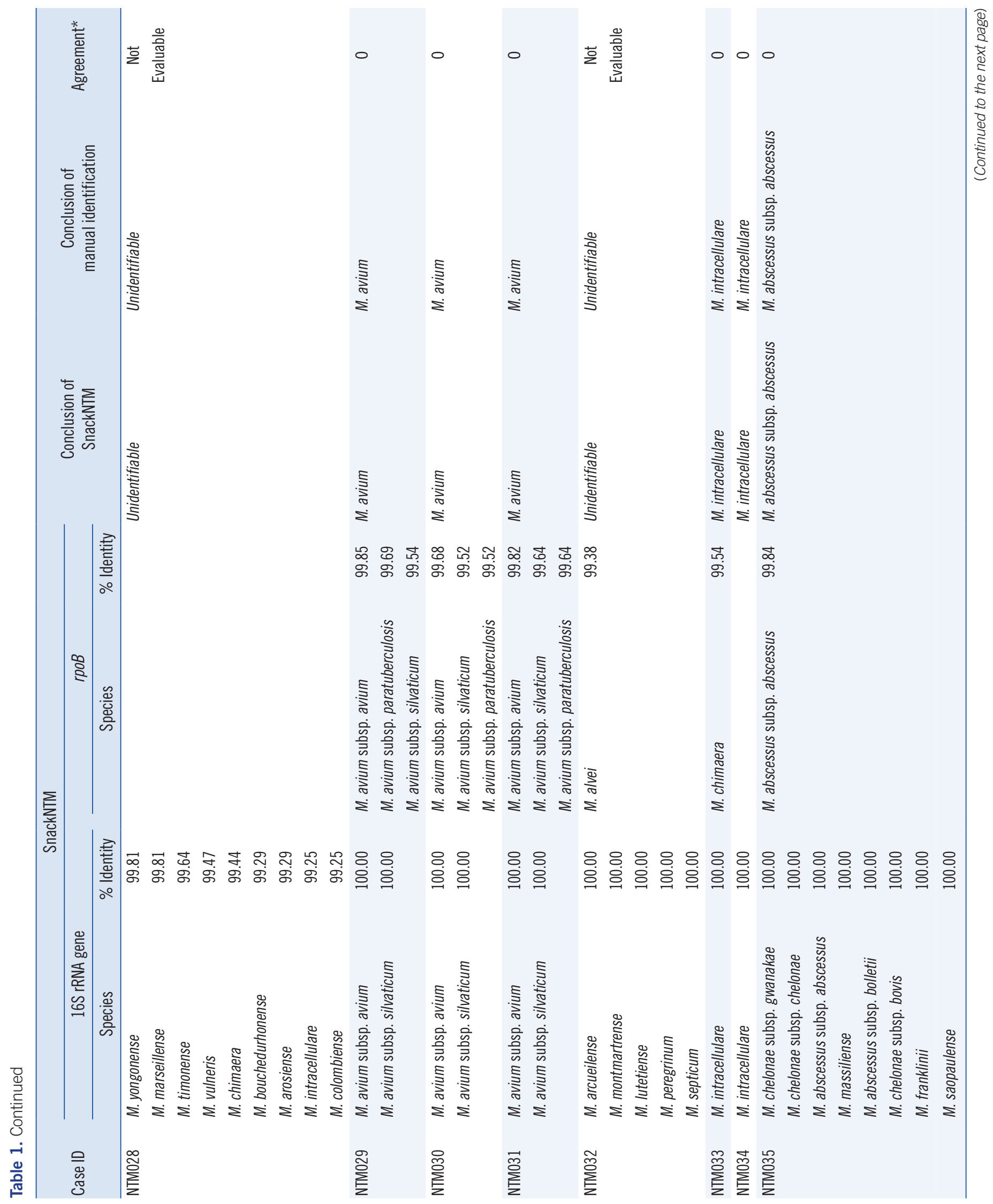




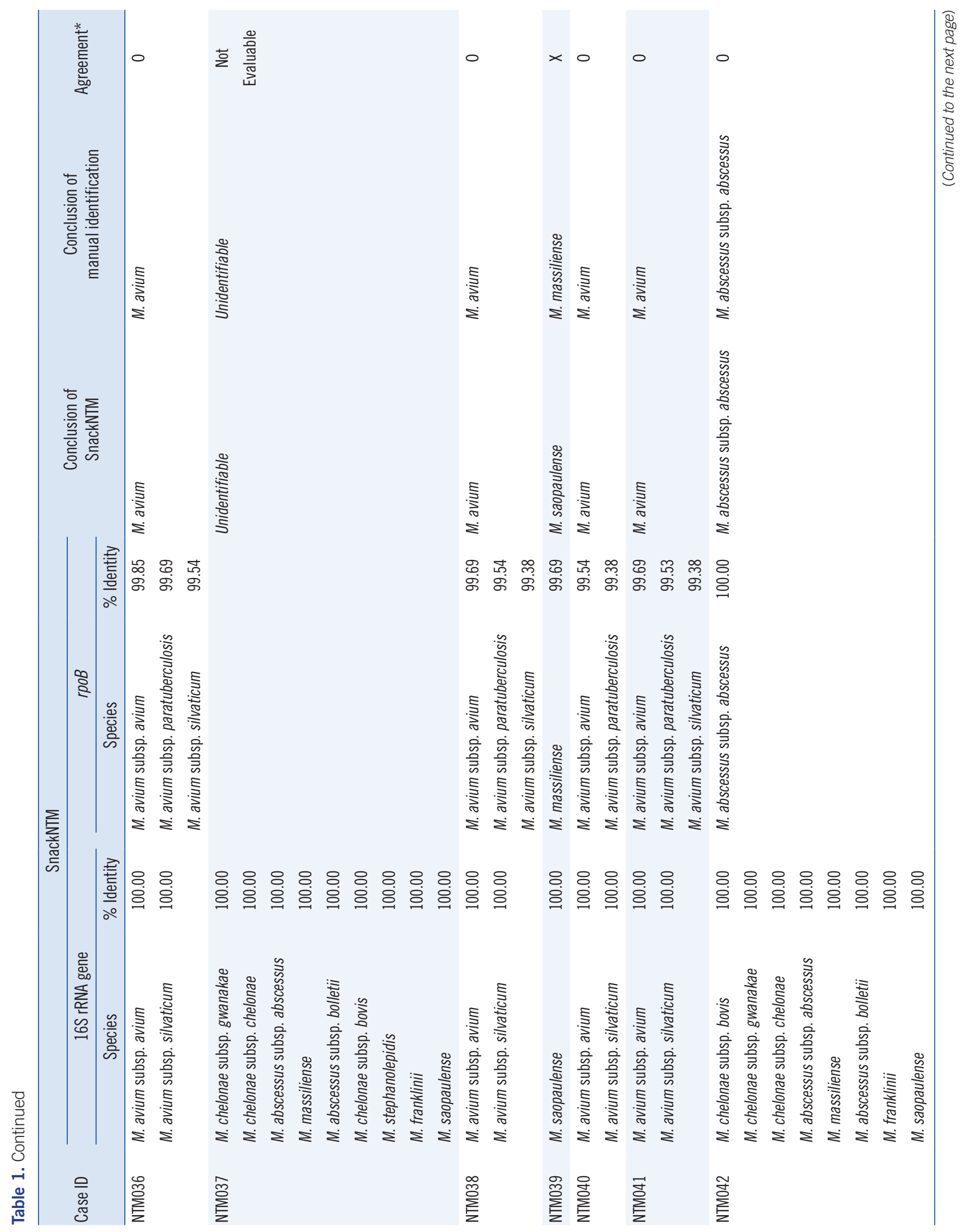




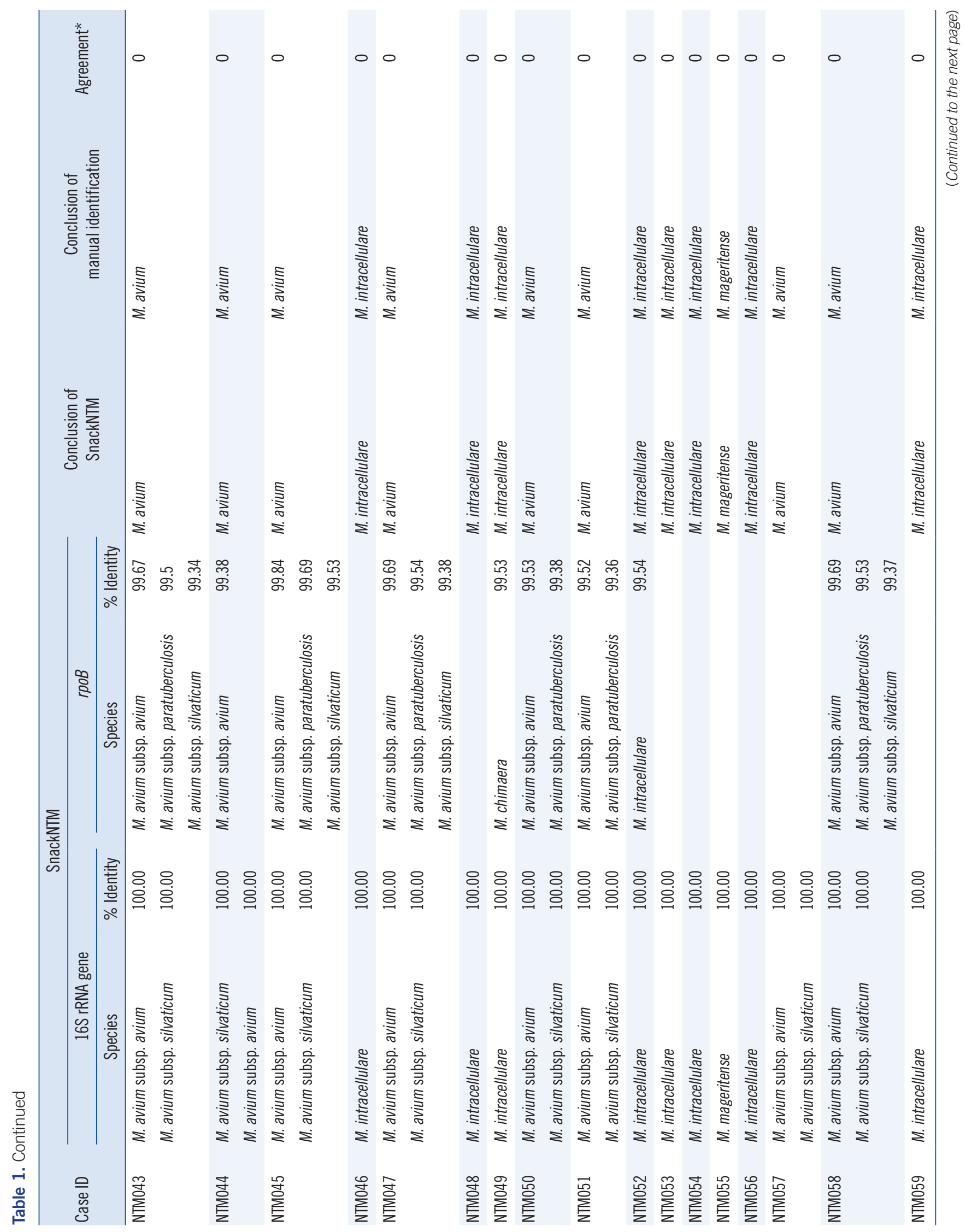


Kim YG, et al.

SnackNTM software for NTM identification

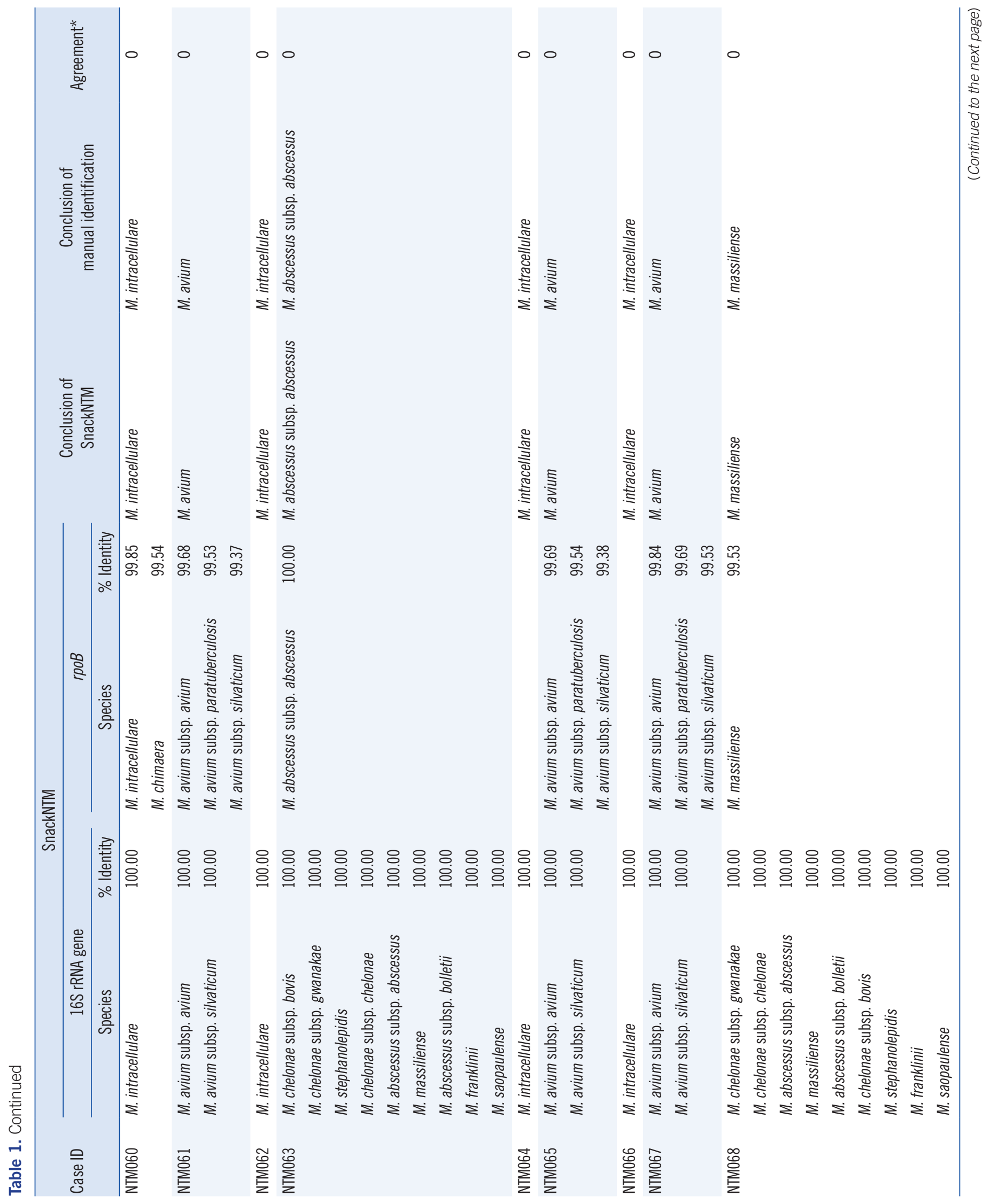




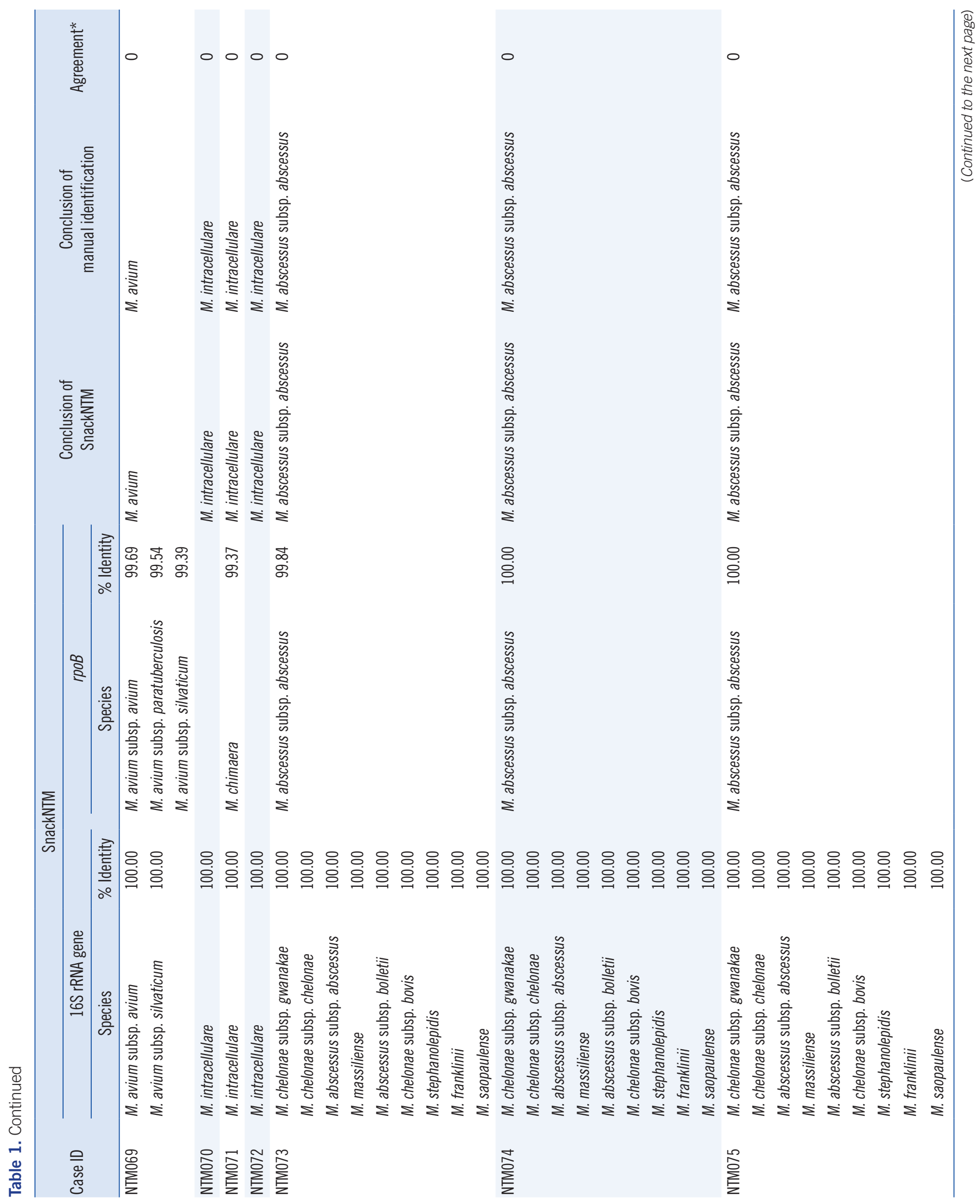


Kim YG, et al.

SnackNTM software for NTM identification

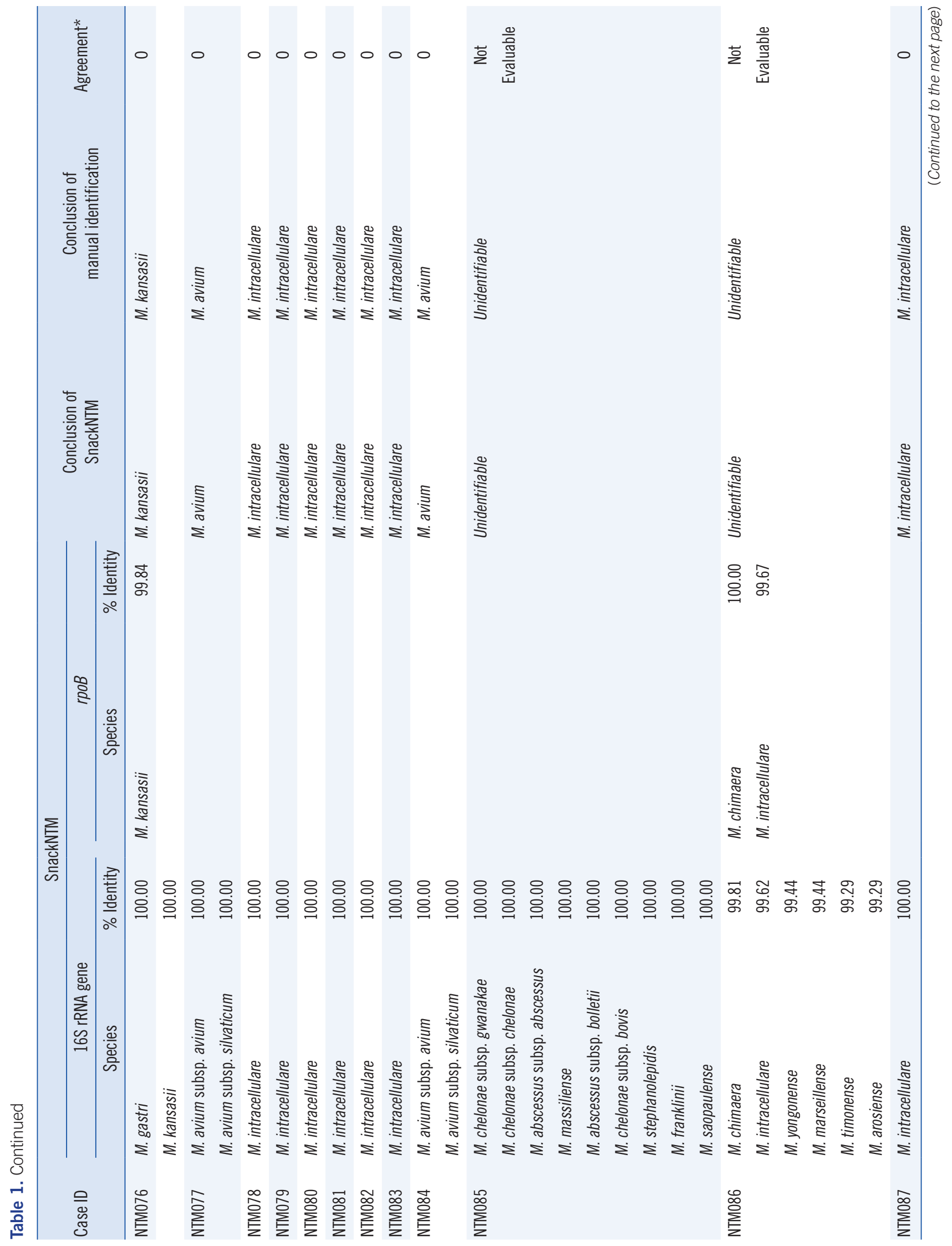




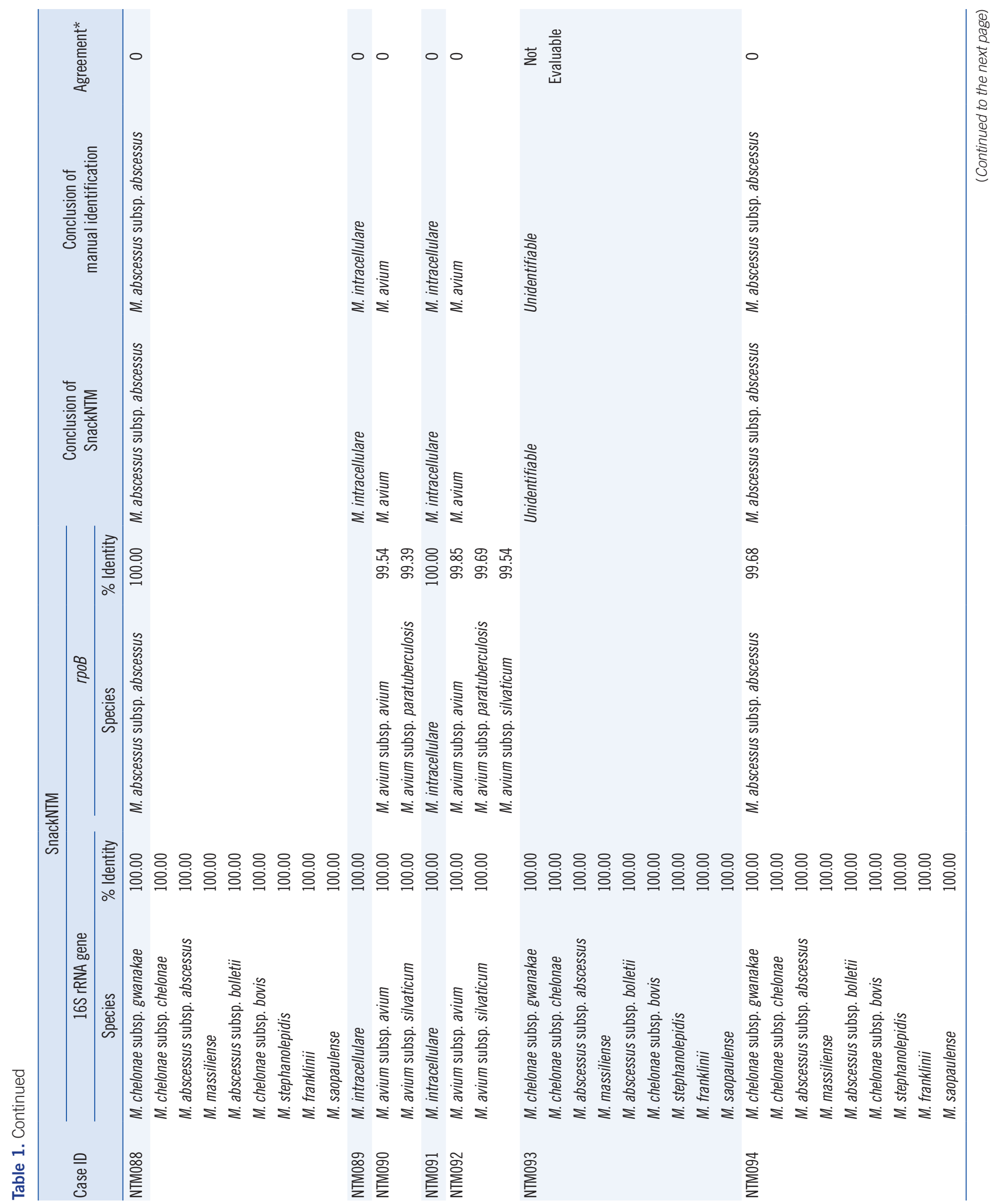




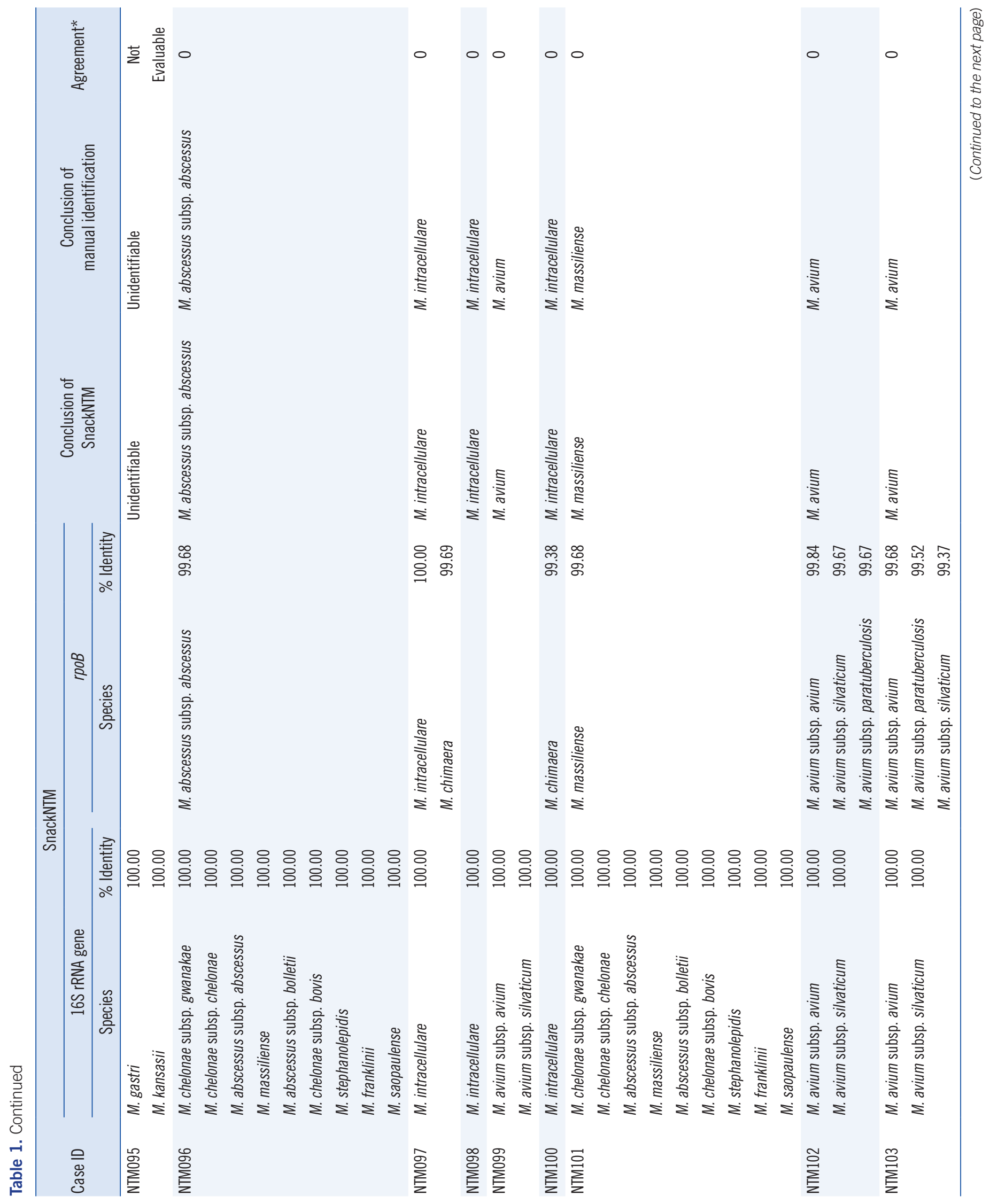




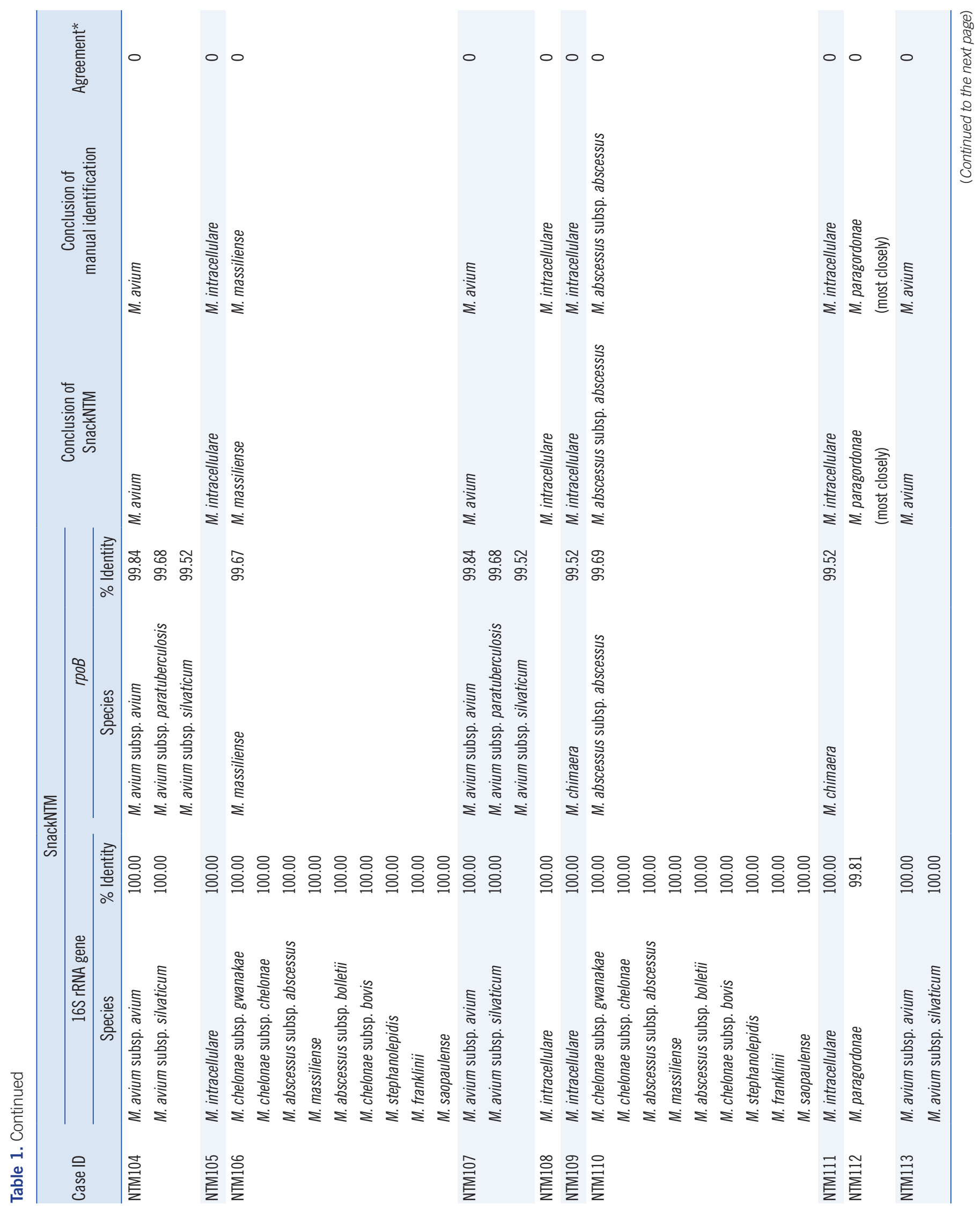


Kim YG, et al.

SnackNTM software for NTM identification

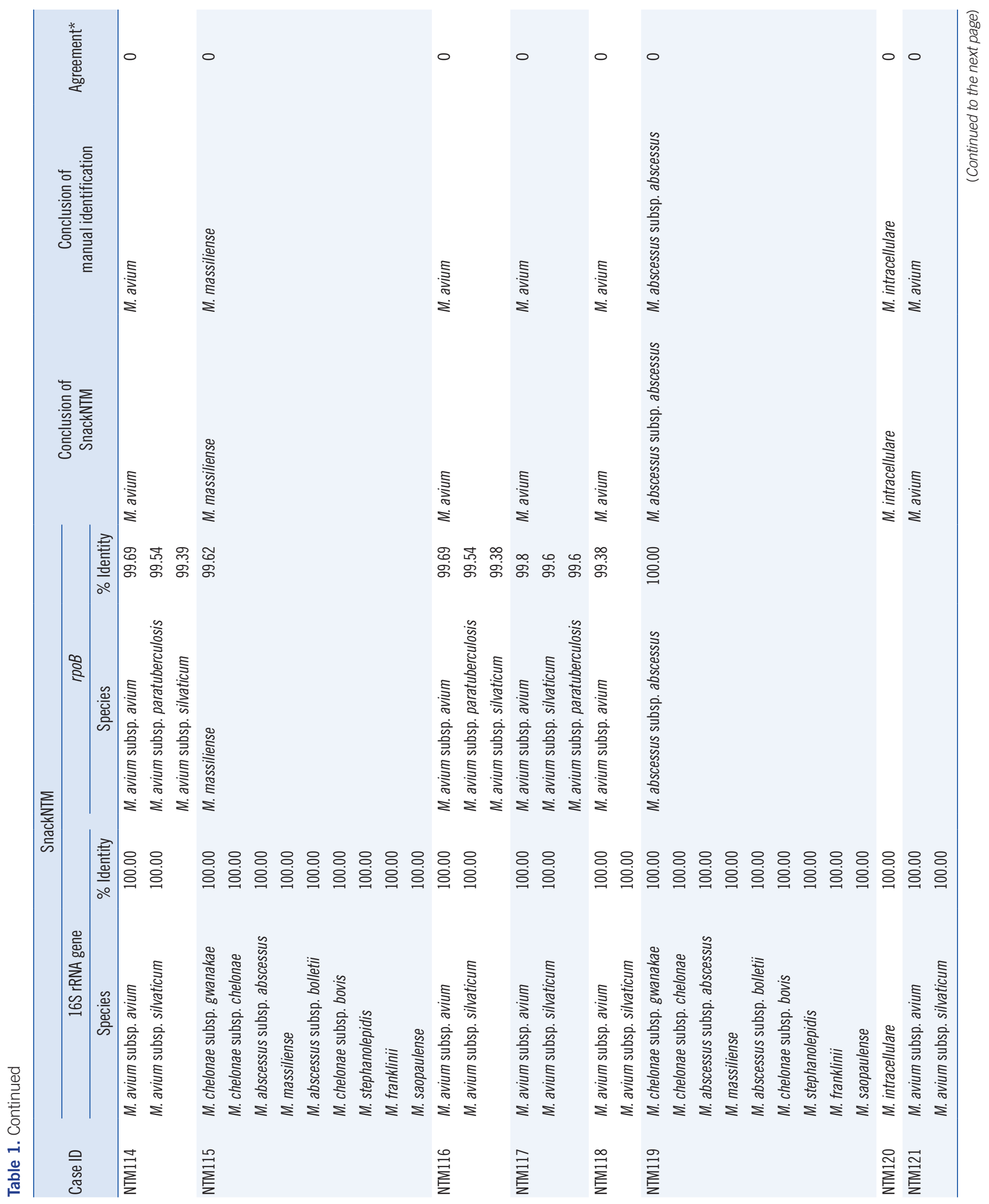




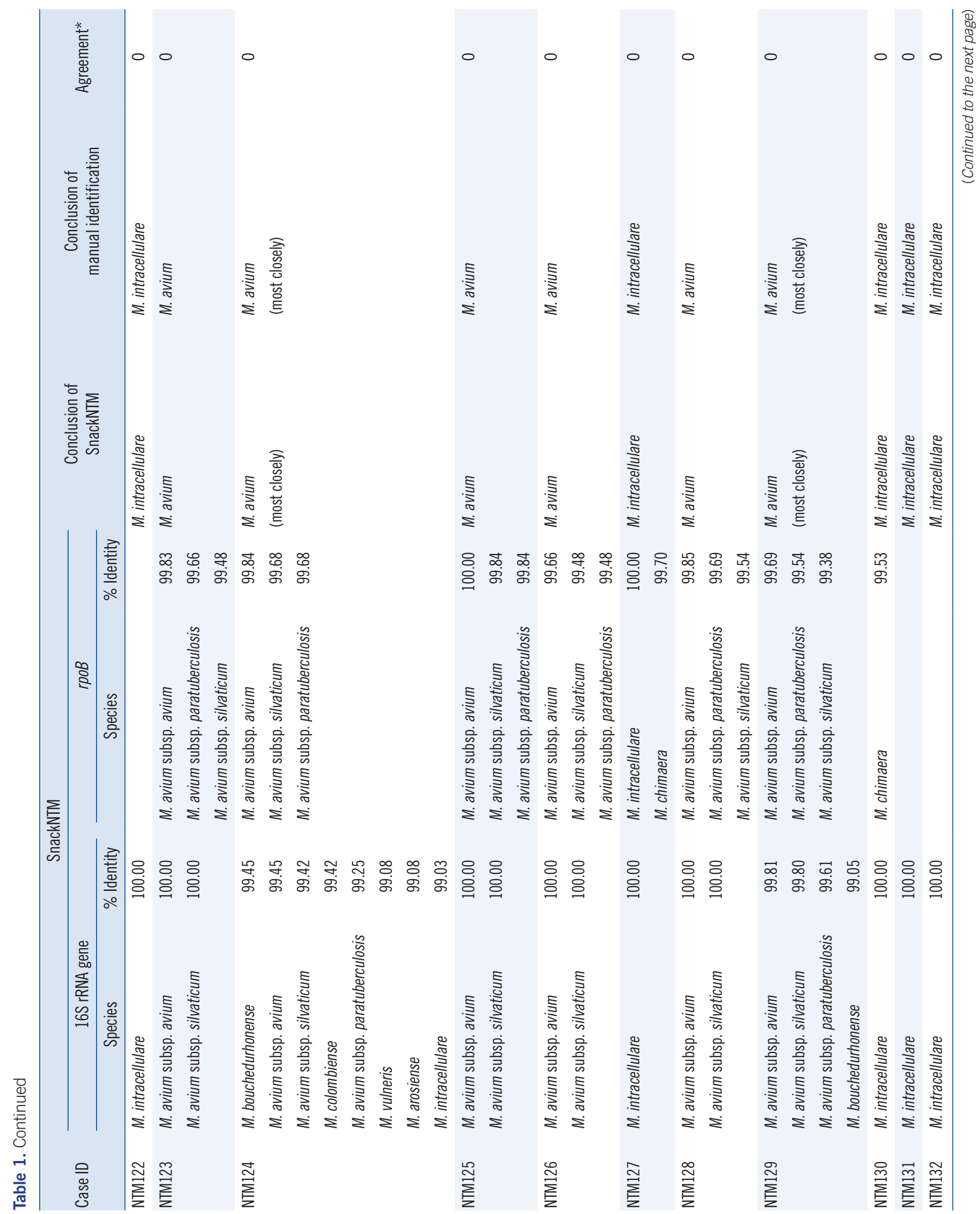


Kim YG, et al.

SnackNTM software for NTM identification

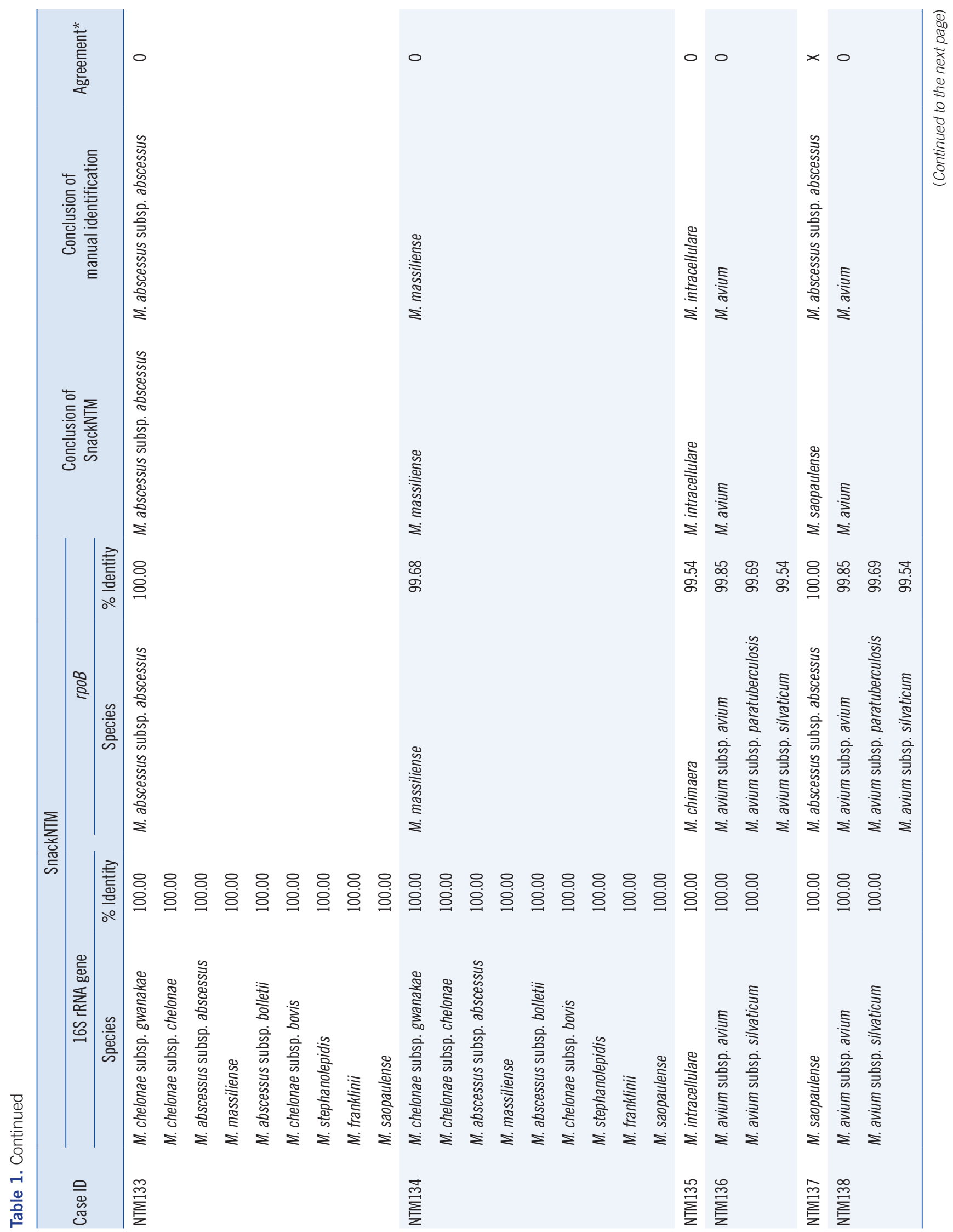




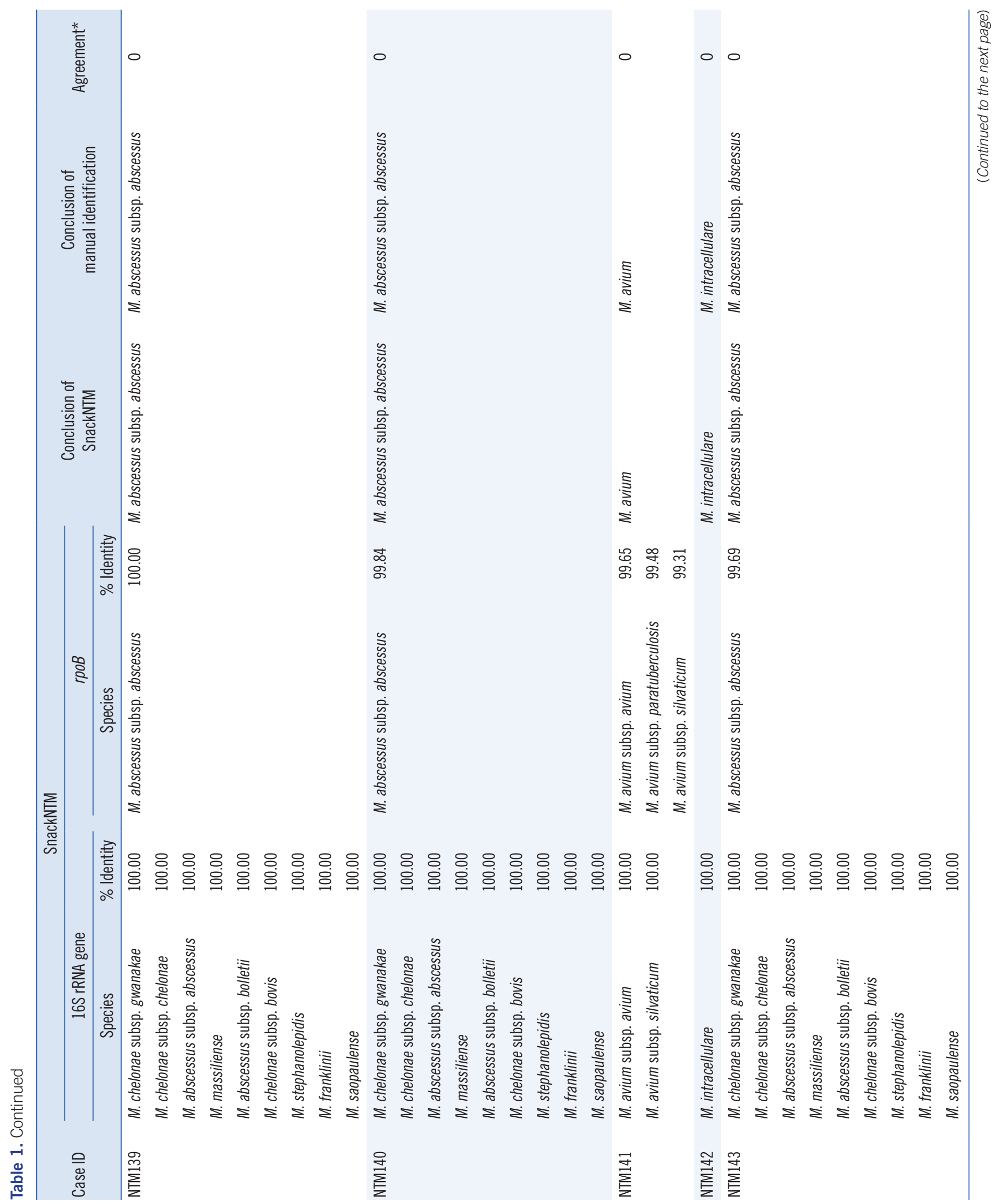


Kim YG, et al.

SnackNTM software for NTM identification

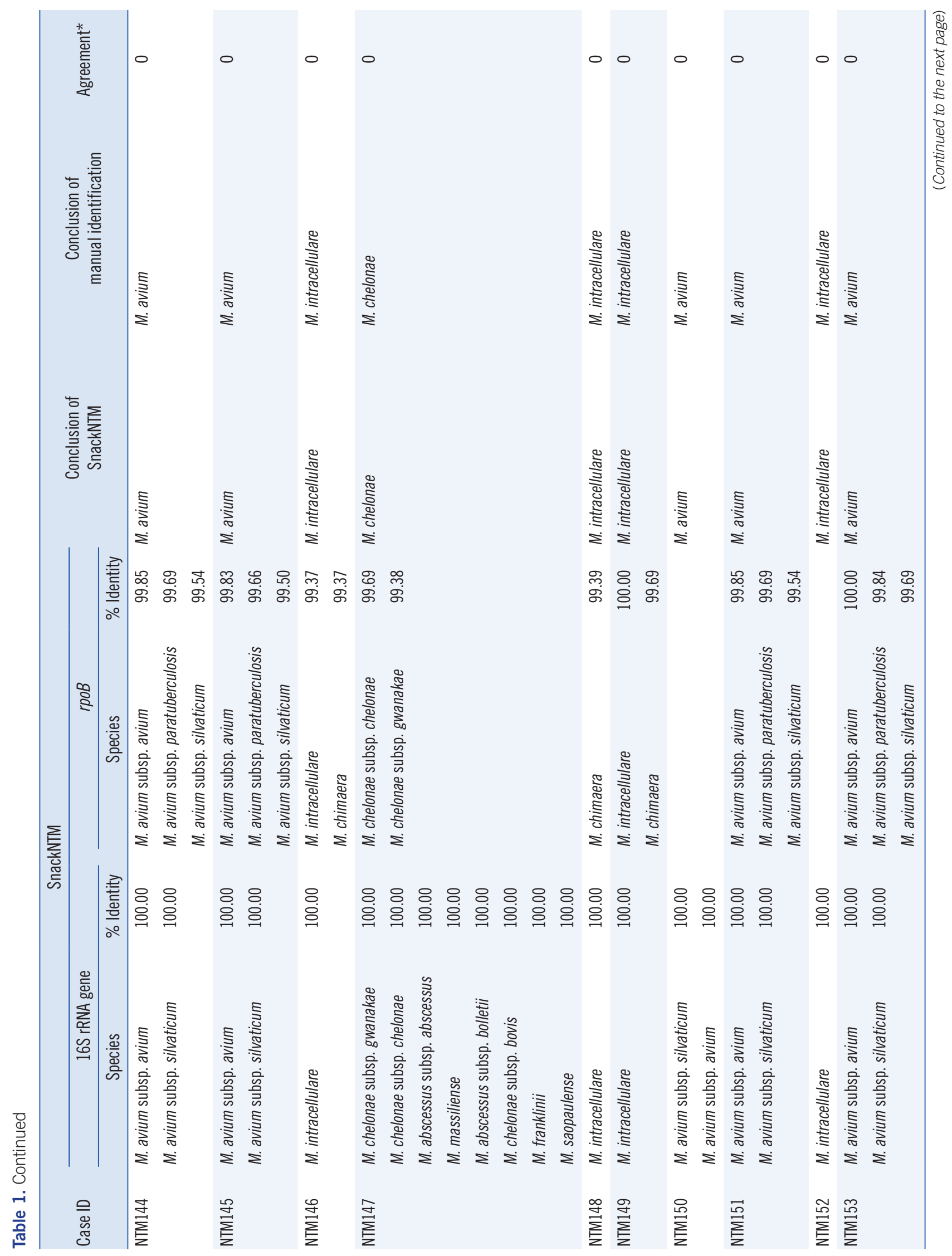




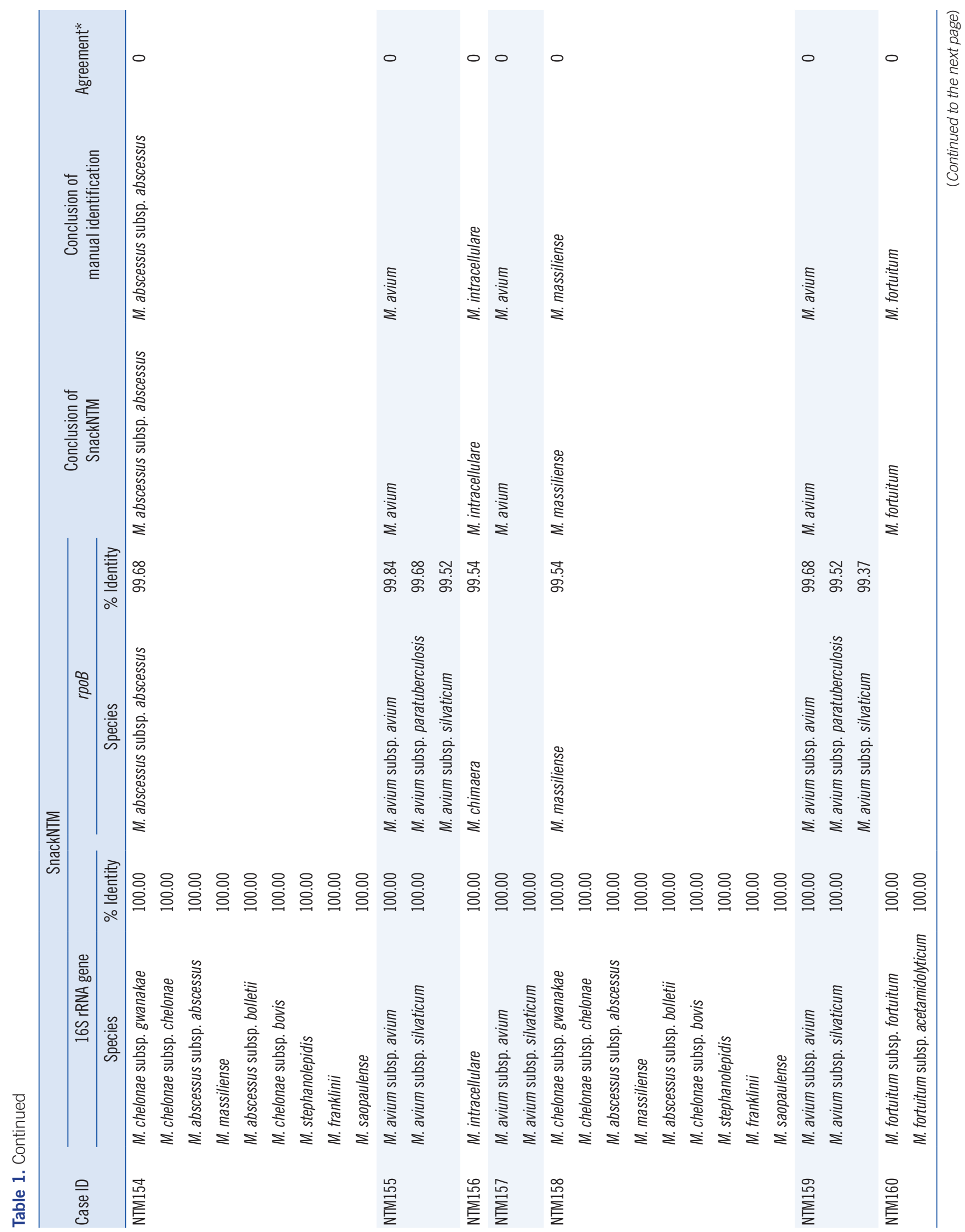


Kim YG, et al.

SnackNTM software for NTM identification

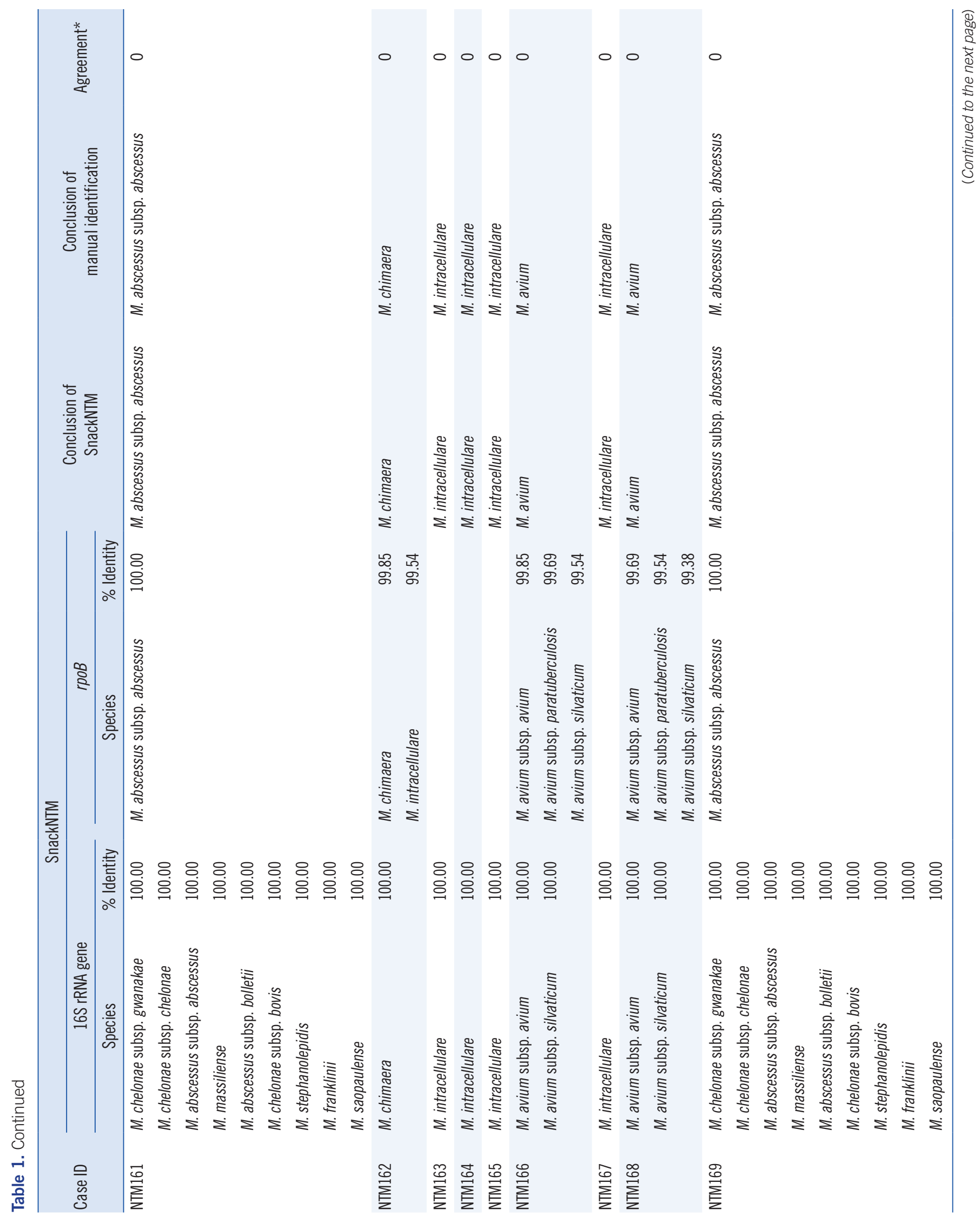




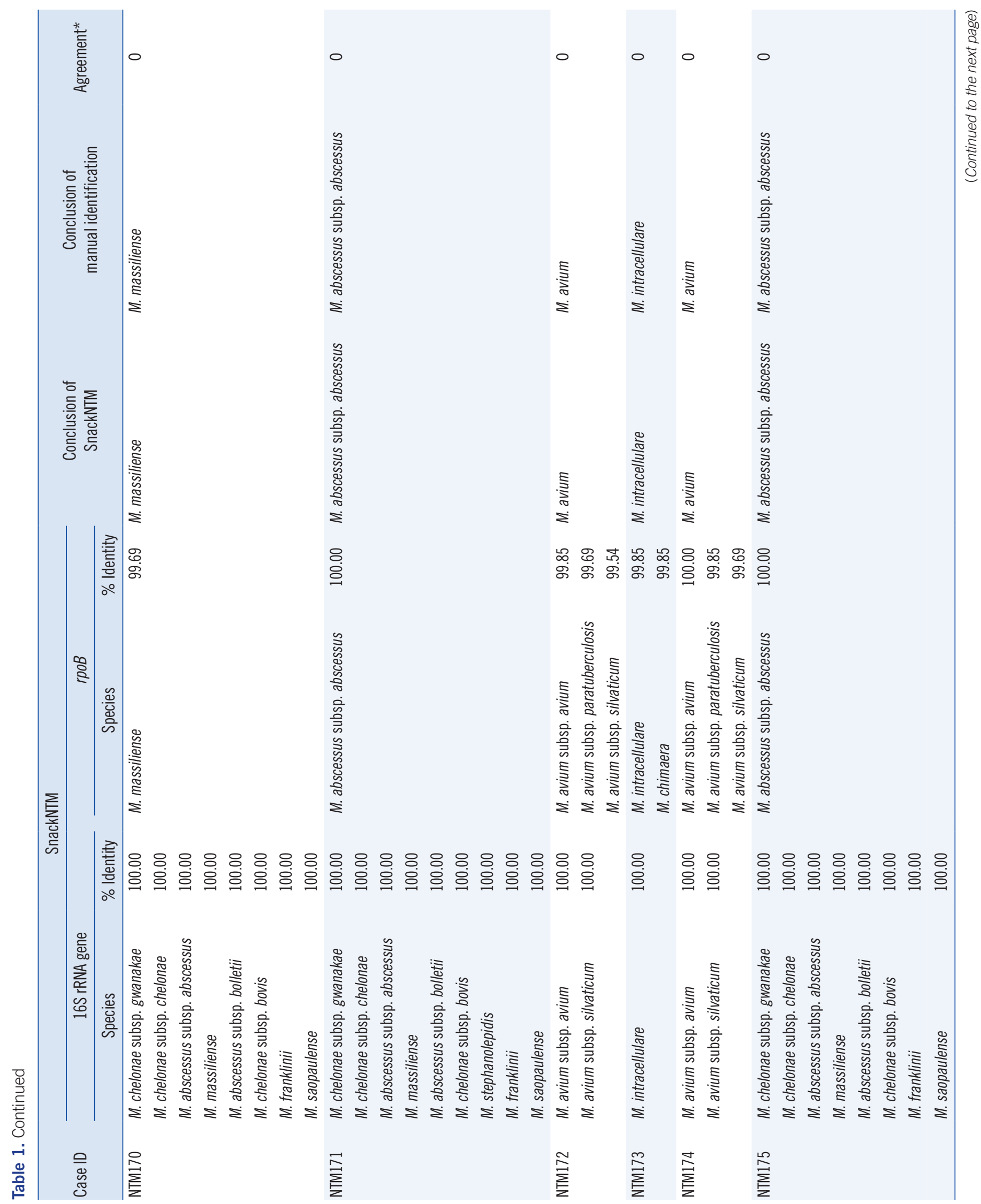


Kim YG, et al.

SnackNTM software for NTM identification

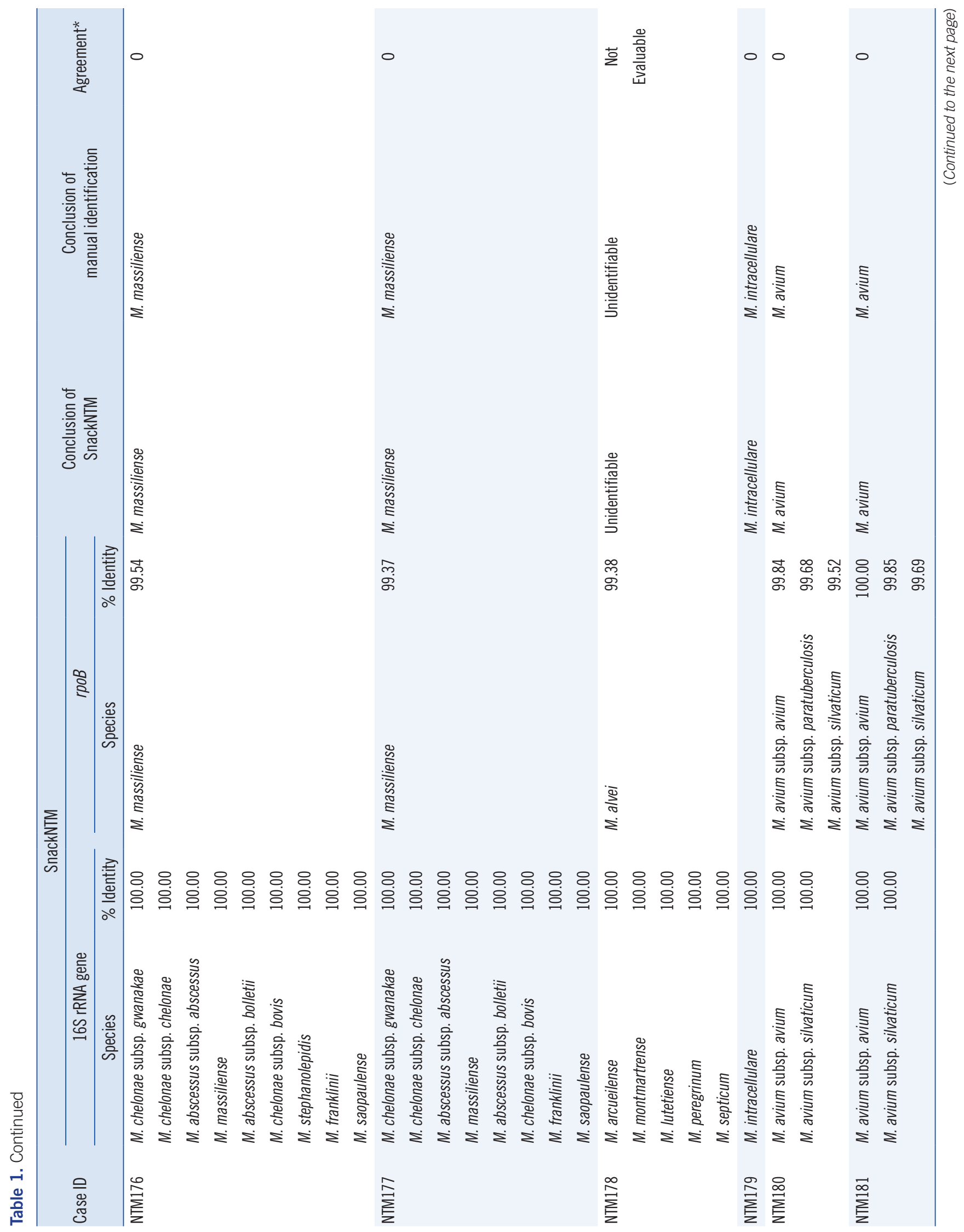




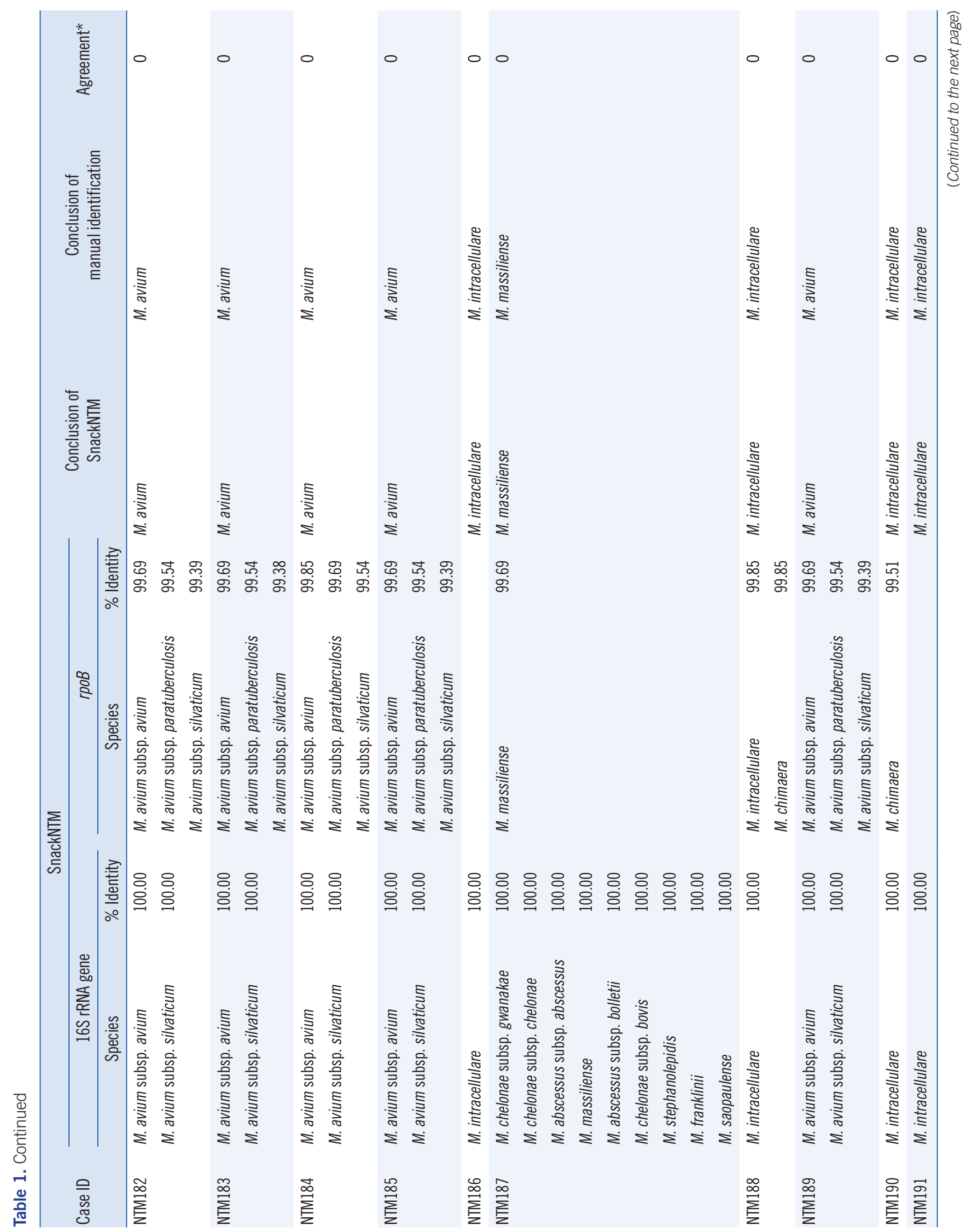


Kim YG, et al.

SnackNTM software for NTM identification

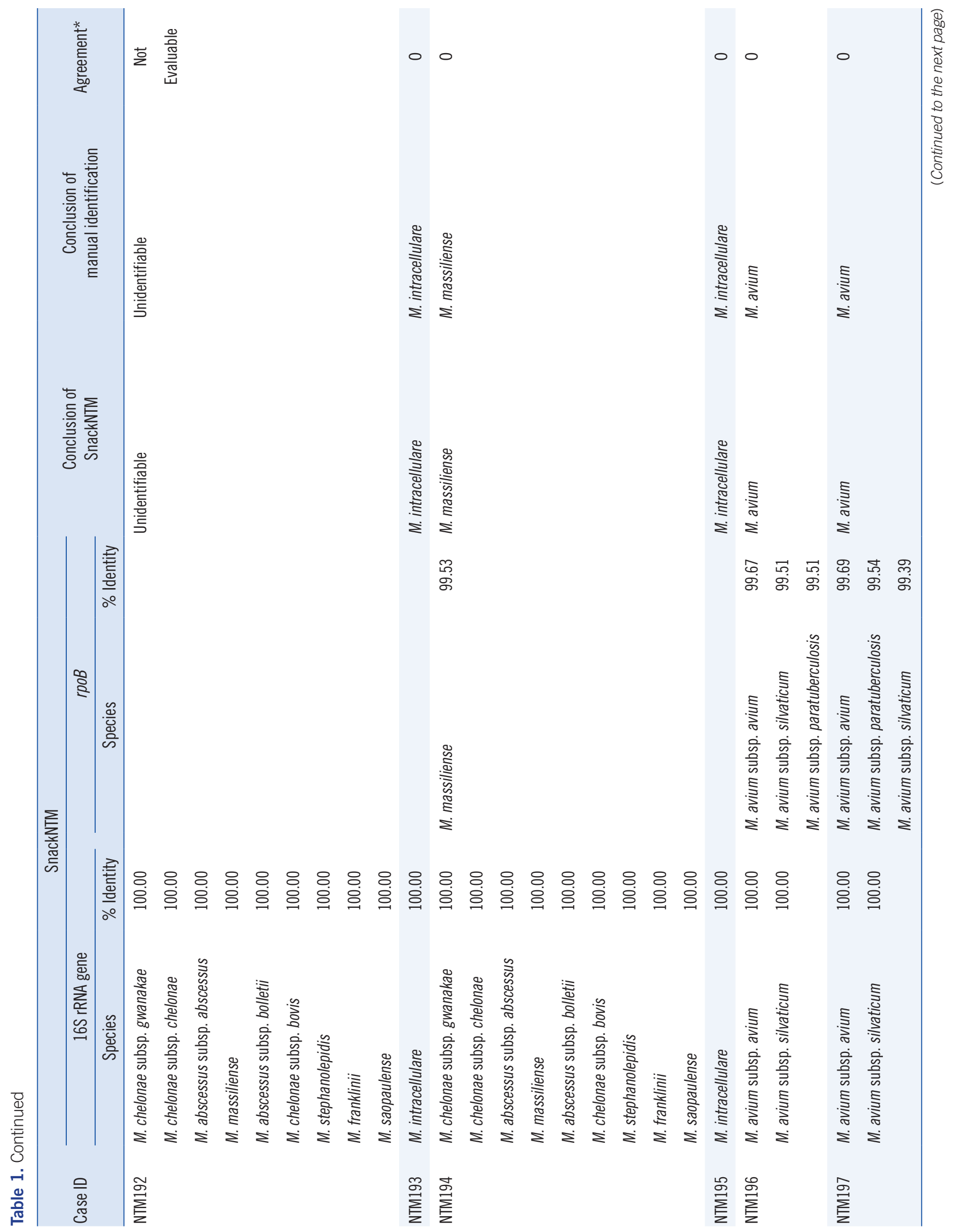




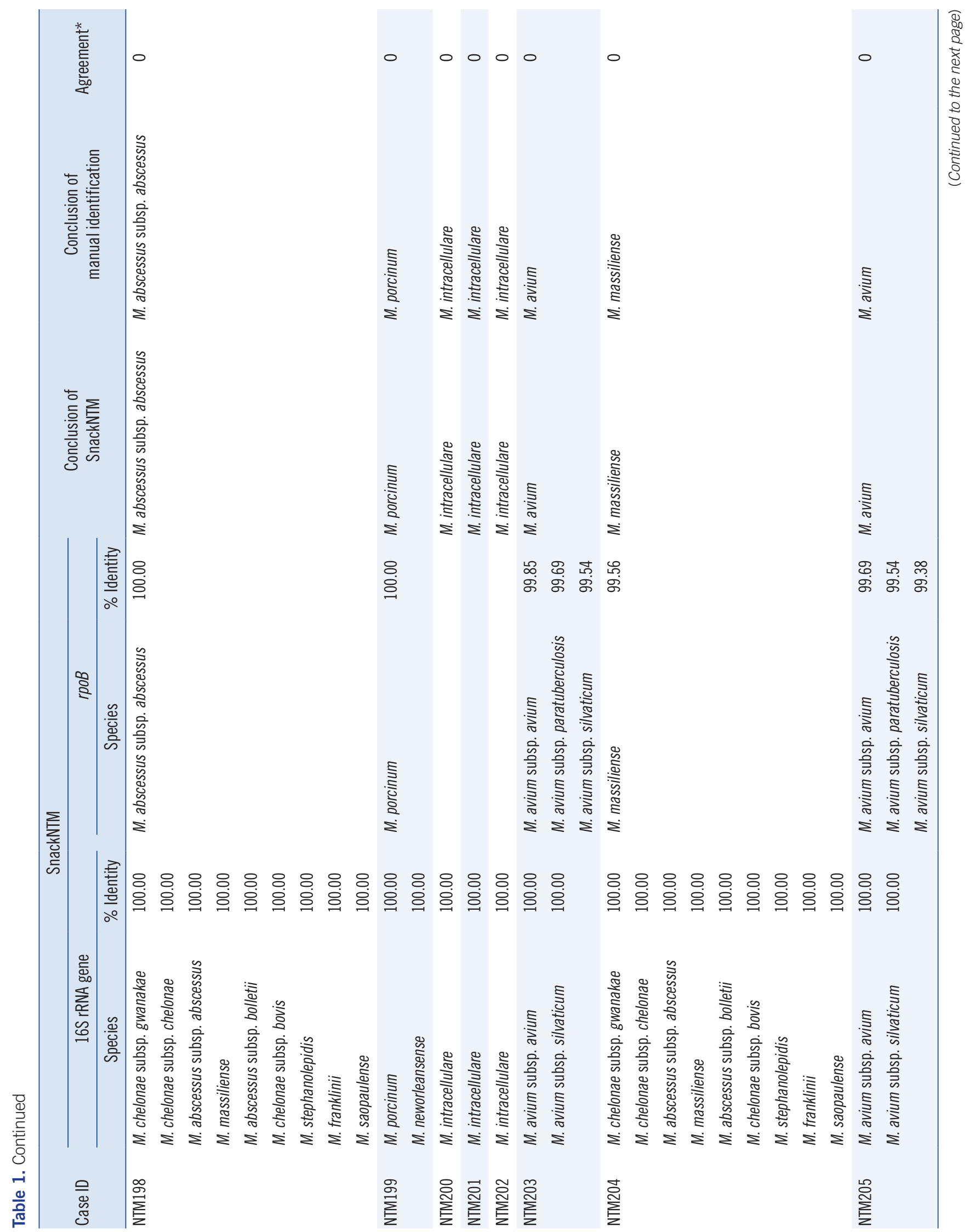


Kim YG, et al.

SnackNTM software for NTM identification

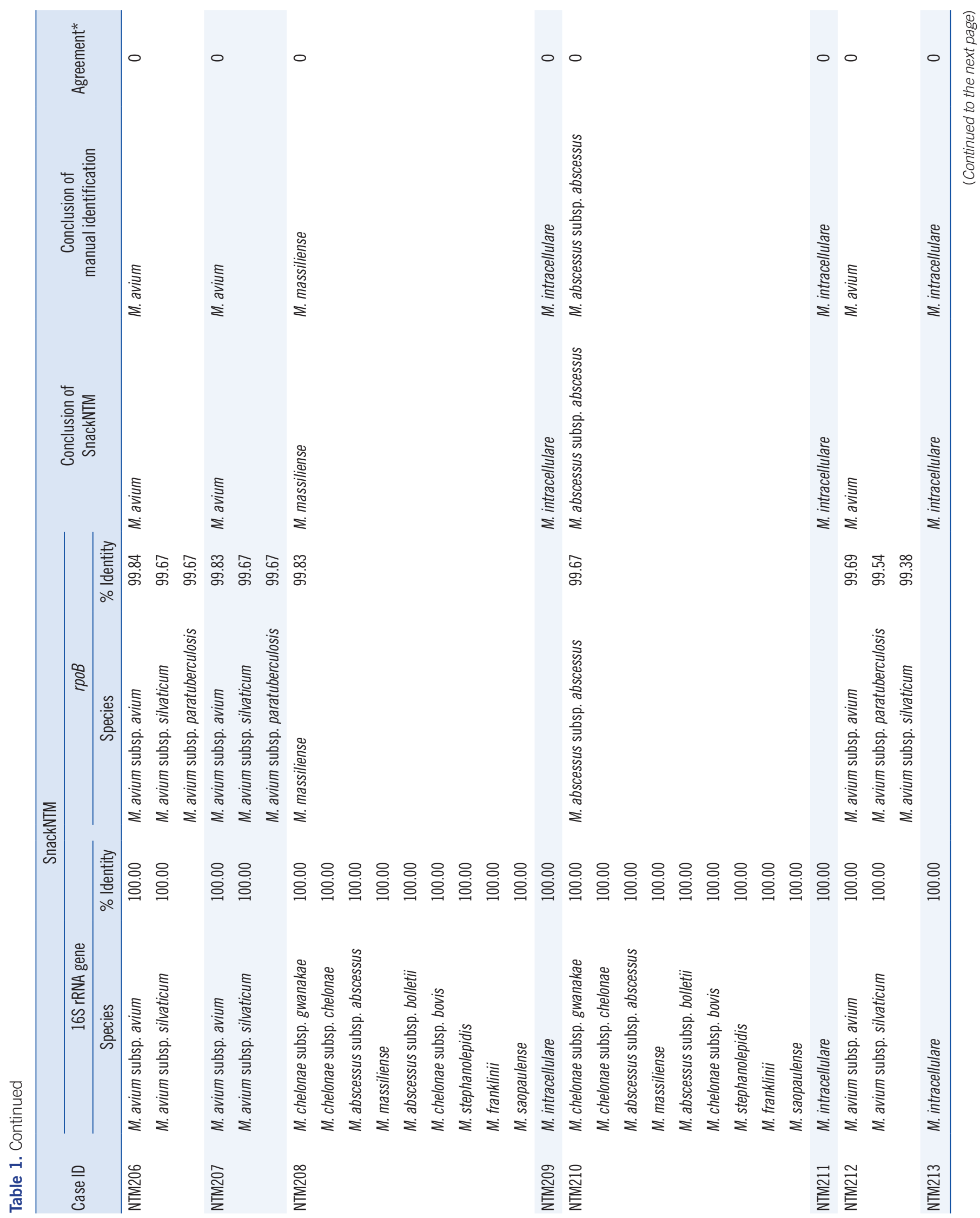




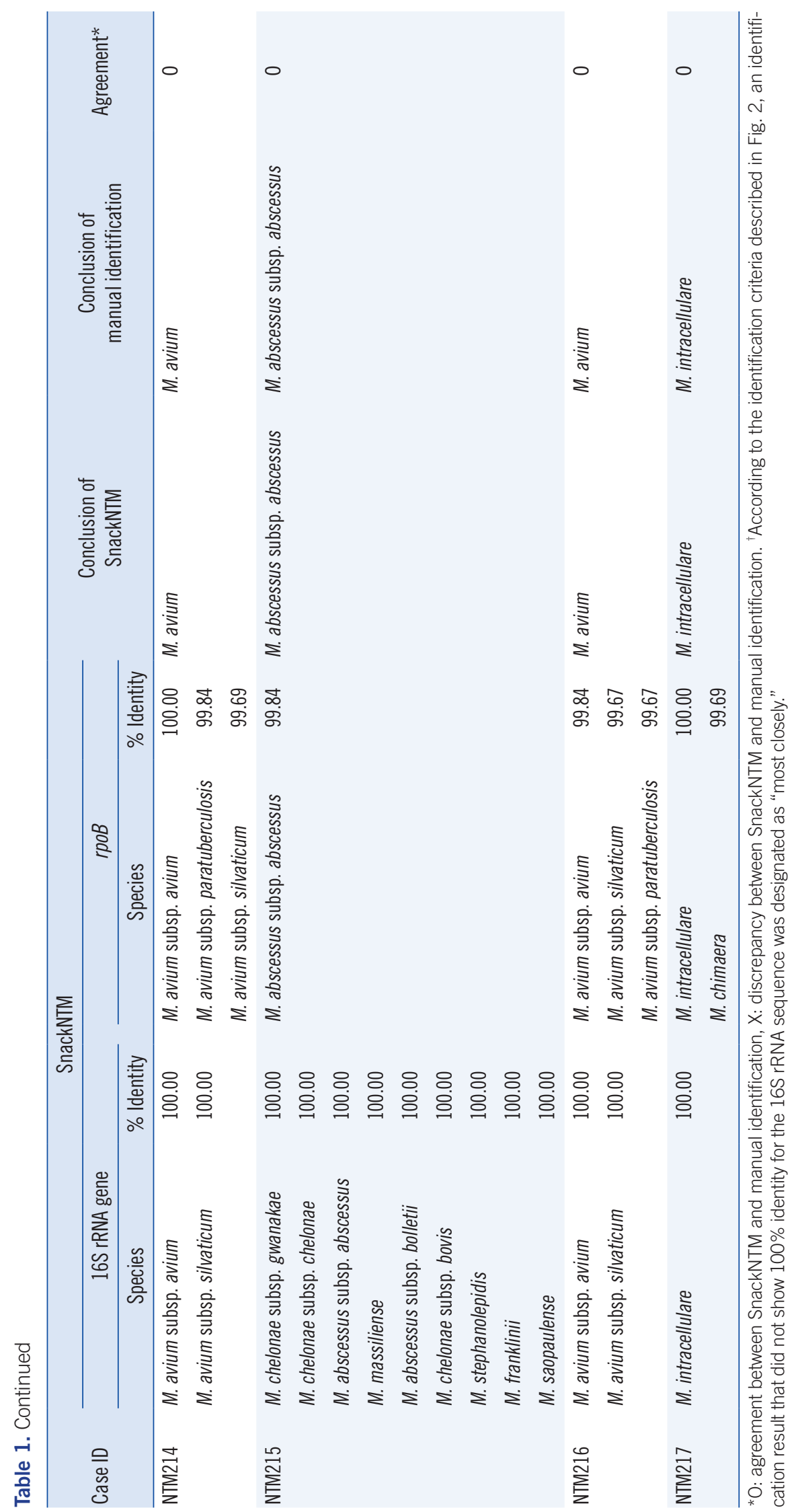


Table 2. Distribution of species identified from consecutive case data using SnackNTM

\begin{tabular}{lc}
\hline Species & Count (\%) \\
\hline M. avium & $80(36.9)$ \\
M. intracellulare & $74(34.1)$ \\
M. abscessus subsp. abscessus & $27(12.4)$ \\
M. massiliense & $16(7.4)$ \\
M. kansasii & $2(0.9)$ \\
M. paragordonae & $2(0.9)$ \\
M. saopaulense* & $2(0.9)$ \\
M. chelonae & $1(0.5)$ \\
M. chimaera & $1(0.5)$ \\
M. fortuitum & $1(0.5)$ \\
M. mageritense & $1(0.5)$ \\
M. porcinum & $1(0.5)$ \\
Unidentifiable & $9(4.1)$ \\
Total & $217(100.0)$ \\
\hline
\end{tabular}

*Two cases that were identified as M. saopaulense, NTM039 and NTM137, showed discrepant results on running SnackNTM and manual identification. By manual identification, NTM039 was identified as M. massiliense, and NTM137 was identified as M. abscessus subsp. abscessus.

150 minutes on average, respectively. The processing time included the time required for writing reports of the 30 cases, which was approximately 30 minutes for both methods. The running time of SnackNTM was approximately 2 minutes, and the remainder of the 10 minutes were mostly spent on reviewing and modifying the results when required. For manual identification, the first part, using Sequencher to review, trim, and align the sequences to produce the input for BLAST, and the second part, comprising running BLAST and interpreting the results, took approximately 60 minutes each.

\section{DISCUSSION}

We developed and validated SnackNTM, a simple and fully automated software for Sanger sequence-based NTM identification. Except for reviewing of the chromatograms generated by the sequencer, all bioinformatics processes involved in sequence-based NTM identification are automatically performed with a single mouse click. The benefit of using SnackNTM is maximized when multiple cases are to be evaluated, as SnackNTM can read multiple trace files at once and sort them according to predefined file naming rules. SnackNTM is free to use and can be instantly used by other laboratories if the primer pairs described herein are used for sequencing. Laboratories can also target different genome regions, if they obtain the appropriate reference sequences.

Searching online databases such as GenBank is considered the standard method for sequence-based identification. However, not all sequences in GenBank are rigorously validated [10-12]. An exhaustive GenBank search usually yields numerous results, including low-quality data, and search filtering options such as "representative genomes only" result in rare species missing from the results. In contrast, SnackNTM contains 16S rRNA gene sequences of all validly published Mycobacterium species in the List of Prokaryotic names with Standing in Nomenclature (LPSN) and rpoB sequences of 174 validly published species in LPSN, curated from GenBank [8]. Sequences of type strains were curated whenever available. In addition, the software has reference sequences saved in FASTA format that can be easily updated by users.

In two cases (NTM039 and NTM137) that showed discrepant SnackNTM and manual identification results, the discrepancy was due to the use of different trimming criteria. In manual identification, shorter fragments of 16S rRNA gene traces were utilized because larger portions of the sequences were trimmed, resulting in $100 \%$ identity to multiple species, including M. saopaulense. A single species was selected based on the rpoB sequences. However, in SnackNTM, longer sequence fragments were included in 16S rRNA gene analysis, resulting in 100\% identity only to $M$. saopaulense and rendering rpoB analysis unnecessary.

Nine cases ( $4.1 \%$ of 217 consecutive cases) were unidentifiable by SnackNTM because the results indicated multiple species. Paradoxically, according to our definition of an unidentifiable result, the more species included in the reference sequence database, the higher the possibility of unidentifiable results. However, in these cases, the unidentifiable result does not necessarily imply identification failure. Additional information, such as the growth rate, photochromogenicity, and incidence in the region, could be utilized to narrow down the species. SnackNTM utilizes a comprehensive set of reference sequences that comprises $>200$ species, and utilizing only two target regions is bound to have some level of unidentifiable results. Attempts have been made to utilize other target regions, such as hsp65 and internal transcribed spacers between the 16S and $23 S$ rRNA genes, for mycobacterial identification [10, 16, 20]. To increase its discriminatory power, additional target regions could be incorporated into SnackNTM in future.

In the unique species data, five cases (17.9\%) yielded different results in SnackNTM and EzBioCloud. Some level of dis- 
Table 3. Identification results of unique species data using SnackNTM and EzBioCloud*

\begin{tabular}{|c|c|c|c|c|c|}
\hline №. case & Case ID & $\begin{array}{l}\text { Manual identification } \\
\text { (16S rRNA gene+rpoB) }\end{array}$ & $\begin{array}{c}\text { SnackNTM } \\
\text { (16S rRNA gene+rpoB) }\end{array}$ & $\begin{array}{c}\text { EzBioCloud } \\
\text { (16S rRNA gene) }\end{array}$ & Cause of discrepancy \\
\hline 1 & NTM001 & M. intracellulare & M. intracellulare & M. intracellulare & \\
\hline 2 & NTM003 & M. kansasii & M. kansasii & M. kansasii & \\
\hline 3 & NTM004 & M. paragordonae & M. paragordonae & M. paragordonae & \\
\hline 4 & NTM008 & M. abscessus subsp. abscessus & M. abscessus subsp. abscessus & M. stephanolepidis & $r p o B$ \\
\hline 5 & NTM009 & M. avium & M. avium & M. avium & \\
\hline 6 & NTM018 & M. massiliense & M. massiliense & M. stephanolepidis & $r p o B$ \\
\hline 7 & NTM055 & M. mageritense & M. mageritense & M. mageritense & \\
\hline 8 & NTM147 & M. chelonae & M. chelonae & M. chelonae & \\
\hline 9 & NTM160 & M. fortuitum & M. fortuitum & M. fortuitum & \\
\hline 10 & NTM162 & M. chimaera & M. chimaera & M. chimaera & \\
\hline 11 & NTM199 & M. porcinum & M. porcinum & M. porcinum & \\
\hline 12 & NTM218 & M. kumamotonense & M. kumamotonense & M. kumamotonense & \\
\hline 13 & NTM219 & M. yongonense & M. yongonense & M. marseillense & $r p o B$ \\
\hline 14 & NTM220 & M. arupense & M. arupense & M. arupense & \\
\hline 15 & NTM221 & M. peregrinum & M. peregrinum & M. montmartrense & $r p o B$ \\
\hline 16 & NTM222 & M. timonense & M. timonense & M. timonense & \\
\hline 17 & NTM223 & M. gordonae & M. gordonae & M. gordonae & \\
\hline 18 & NTM224 & M. neoaurum & M. neoaurum & M. neoaurum & \\
\hline 19 & NTM225 & M. septicum & M. septicum & M. montmartrense & \\
\hline 20 & NTM226 & M. marseillense & M. marseillense & M. marseillense & \\
\hline 21 & NTM227 & M. mucogenicum & M. mucogenicum & M. mucogenicum & \\
\hline 22 & NTM228 & M. parascrofulaceum & M. parascrofulaceum & M. parascrofulaceum & \\
\hline 23 & NTM229 & M. lentiflavum & M. Ientiflavum & M. lentiflavum & \\
\hline 24 & NTM230 & M. haemophilum & M. haemophilum & M. haemophilum & \\
\hline 25 & NTM231 & M. brisbanense & M. brisbanense & M. dioxanotrophicus & Different set of reference sequences \\
\hline 26 & NTM232 & M. xenopi & M. xenopi & M. xenopi & \\
\hline 27 & NTM233 & M. angelicum & M. angelicum & M. szulgai & \\
\hline 28 & NTM234 & M. persicum & M. persicum & M. persicum & \\
\hline
\end{tabular}

*https://www.ezbiocloud.net/identify.

crepancy was expected because of the difference in target regions, i.e., the $16 \mathrm{~S}$ rRNA gene and $r p o B$ in SnackNTM vs. the $16 \mathrm{~S}$ rRNA gene in EzBioCloud. In the majority of cases (4/5, $80.0 \%$ ) the discrepancy was caused by the utilization of rpoB in SnackNTM. In these cases, there were multiple species with the same identity score based on 16S rRNA gene sequences. SnackNTM narrows down to a single species based on rpoB sequence analysis, whereas EzBioCloud provides a single species based on their own criteria. The EzBioCloud result for NTM231 was $M$. dioxanotrophicus, which was not included in the reference sequence database of SnackNTM. The nomenclatural status of M. dioxanotrophicus in LPSN was "not validly published" at the time of writing.

This study had several limitations. First, Sanger sequencing of the two target regions we used, i.e., the 16S rRNA gene and $r p o B$, was not the only and may not be the best method for NTM identification. In the lack of a consensus, the decision to use these two targets was based on practical considerations for clinical use, such as cost-effectiveness and available reference sequences, rather than a strong scientific ground. In addition, conventional methods, such as line probe assays, have their own benefits, and emerging methods, such as MALDI-TOF MS, are promising in the field of NTM identification [2, 19, 21]. Second, SnackNTM was validated based on experiments per- 
formed using sequencing data files, not real samples. As a result, only the bioinformatics process of SnackNTM, not the entire identification process, could be validated. As we used the same set of reference sequences for SnackNTM and manual identification, the results of the two methods were expected to be identical, except for the discrepancies, which were due to different trimming criteria.

In conclusion, SnackNTM represents an efficient software for the automation of Sanger sequence-based NTM species identification. As there is no single authorized and validated database of reference sequences of all published mycobacteria, the laboratory database of SNUH was incorporated into SnackNTM. SnackNTM is free to use, and if required, target regions and reference sequences can be optimized for individual users. SnackNTM is expected to reduce the workload required for Sanger sequence-based NTM identification.

\section{ACKNOWLEDGEMENTS}

None.

\section{AUTHOR CONTRIBUTIONS}

Kim YG developed the software and drafted the manuscript. Jung $\mathrm{K}$ and Kim S performed the experiments and analyzed the data. Kim MJ and Lee JS interpreted the data and contributed to manuscript revision. Park SS and Seong MW supervised the study and performed the final manuscript revision.

\section{CONFLICTS OF INTEREST}

No potential conflicts of interest relevant to this article were reported.

\section{RESEARCH FUNDING}

None declared.

\section{ORCID}

\section{Young-Gon Kim \\ Kiwook Jung \\ Seunghwan Kim \\ Man Jin Kim \\ Jee-Soo Lee \\ Sung-Sup Park}

Moon-Woo Seong

https://orcid.org/0000-0003-2954-3677

\section{REFERENCES}

1. Adjemian J, Daniel-Wayman S, Ricotta E, Prevots DR. Epidemiology of nontuberculous mycobacteriosis. Semin Respir Crit Care Med 2018; 39:325-35.

2. Cowman S, van Ingen J, Griffith DE, Loebinger MR. Non-tuberculous mycobacterial pulmonary disease. Eur Respir J 2019;54:1900250.

3. van Ingen J, Bendien SA, De Lange WC, Hoefsloot W, Dekhuijzen PN, Boeree MJ, et al. Clinical relevance of non-tuberculous mycobacteria isolated in the Nijmegen-Arnhem region, The Netherlands. Thorax 2009; 64:502-6.

4. Aksamit TR, Philley JV, Griffith DE. Nontuberculous mycobacterial (NTM) lung disease: the top ten essentials. Respir Med 2014;108:41725.

5. de Zwaan R, van Ingen J, van Soolingen D. Utility of rpoB gene sequencing for identification of nontuberculous mycobacteria in the Netherlands. J Clin Microbiol 2014;52:2544-51.

6. Durão V, Silva A, Macedo R, Durão P, Santos-Silva A, Duarte R. Portuguese in vitro antibiotic susceptibilities favor current nontuberculous mycobacteria treatment guidelines. Pulmonology 2019;25:162-7.

7. Stout JE, Koh WJ, Yew WW. Update on pulmonary disease due to nontuberculous mycobacteria. Int J Infect Dis 2016;45:123-34.

8. Parte AC, Carbasse JS, Meier-Kolthoff JP, Reimer LC, Göker M. List of Prokaryotic names with Standing in Nomenclature (LPSN) moves to the DSMZ. Int J Syst Evol Microbiol 2020;70:5607-12.

9. CLSI. Laboratory detection and identification of mycobacteria. 2nd ed. CLSI M48. Wayne, PA: Clinical and Laboratory Standards Institute, 2018.

10. Joao I, Cristovao P, Antunes L, Nunes B, Jordao L. Identification of nontuberculous mycobacteria by partial gene sequencing and public databases. Int J Mycobacteriol 2014;3:144-51.

11. CLSI. Interpretive criteria for identification of bacteria and fungi by targeted DNA sequencing. 2nd ed. CLSI MM18. Wayne, PA: Clinical and Laboratory Standards Institute, 2018.

12. Tortoli E. Standard operating procedure for optimal identification of mycobacteria using 16S rRNA gene sequences. Stand Genomic Sci 2010; 3:145-52.

13. Kim YG, Kim MJ, Lee JS, Lee JA, Song JY, Cho SI, et al. SnackVar: an open-source software for Sanger sequencing analysis optimized for clinical use. J Mol Diagn 2021;23:140-8.

14. Jorgensen JH, Pfaller MA, et al. Manual of clinical microbiology. 11th ed. Washington DC: ASM Press, 2015:579-80.

15. Yamaba Y, Takakuwa O, Saito M, Kawae D, Yoshihara M, Mori Y, et al. Pulmonary Mycobacterium abscessus subspecies abscessus disease that showed a discrepancy between the genotype and phenotype of clarithromycin resistance. Intern Med 2019;58:2675-8.

16. Huang WC, Yu MC, Huang YW. Identification and drug susceptibility testing for nontuberculous mycobacteria. J Formos Med Assoc 2020; 119:S32-41.

17. Kim YG, Lee HY, Kwak NW, Park JH, Kim TS, Kim MJ, et al. Determination of clinical characteristics of Mycobacterium kansasii-derived species by reanalysis of isolates formerly reported as $M$. kansasii. Ann Lab Med 2021;41:463-8.

18. Schweickert B, Goldenberg O, Richter E, Göbel UB, Petrich A, Buchholz $P$, et al. Occurrence and clinical relevance of Mycobacterium chimaera sp. nov., Germany. Emerg Infect Dis 2008;14:1443-6. 
19. Tortoli E, Rindi L, Garcia MJ, Chiaradonna P, Dei R, Garzelli C, et al. Proposal to elevate the genetic variant MAC-A, included in the Mycobacterium avium complex, to species rank as Mycobacterium chimaera sp. nov. Int J Syst Evol Microbiol 2004;54:1277-85.

20. Lecorche E, Haenn S, Mougari F, Kumanski S, Veziris N, Benmansour H, et al. Comparison of methods available for identification of Mycobacteri- um chimaera. Clin Microbiol Infect 2018;24:409-13.

21. Kalaiarasan E, Thangavelu K, Krishnapriya K, Muthuraj M, Jose M, Joseph NM. Diagnostic performance of real time PCR and MALDI-TOF in the detection of nontuberculous mycobacteria from clinical isolates. Tuberculosis (Edinb) 2020;125:101988. 